REPRESENTATION THEORY

An Electronic Journal of the American Mathematical Society

Volume 17, Pages 442-468 (August 5, 2013)

S $1088-4165(2013) 00438-7$

\title{
RANK 2 AFFINE MV POLYTOPES
}

\author{
PIERRE BAUMANN, THOMAS DUNLAP, JOEL KAMNITZER, AND PETER TINGLEY
}

\begin{abstract}
We give a realization of the crystal $B(-\infty)$ for $\widehat{\mathrm{sl}}_{2}$ using decorated polygons. The construction and proof are combinatorial, making use of Kashiwara and Saito's characterization of $B(-\infty)$, in terms of the * involution. The polygons we use have combinatorial properties suggesting they are the $\widehat{\mathrm{sl}}_{2}$ analogues of the Mirković-Vilonen polytopes defined by Anderson and the third author in finite type. Using Kashiwara's similarity of crystals we also give MV polytopes for $A_{2}^{(2)}$, the other rank 2 affine Kac-Moody algebra.
\end{abstract}

\section{INTRODUCTION}

Fix a symmetrizable Kac-Moody algebra $\mathfrak{g}$. Kashiwara's theory of crystals constructs combinatorial data (a set $B(-\lambda)$ along with some operations) corresponding to each integrable lowest weight representation $V(-\lambda)$, which records certain leading order behavior of the representation. The crystals $B(-\lambda)$ form a directed system whose limit $B(-\infty)$ can be thought of as the crystal for $U^{+}(\mathfrak{g})$. This theory makes heavy use of the quantized universal enveloping algebra $U_{q}(\mathfrak{g})$ associated with $\mathfrak{g}$, but there are also combinatorial characterizations of $B(-\infty)$, and it can often be realized by more elementary means.

When $\mathfrak{g}$ is of finite type, there is a realization of $B(-\infty)$ using Mirković-Vilonen (MV) polytopes, developed by Anderson [1] and the third author [8, 9]. In this paper, we realize $B(-\infty)$ for $\widehat{\mathrm{sl}}_{2}$ using a collection of decorated polytopes in the $\widehat{\mathrm{sl}}_{2}$ root lattice. Our construction has many properties in common with finite type, which we take as evidence that we have found the correct generalization of MV polytopes in type $\widehat{\mathrm{sl}}_{2}$ :

(i) An MV polytope is uniquely determined by either its right or left side, and any candidate for one side does correspond to an MV polytope.

(ii) Kashiwara's involution on $B(-\infty)$ is negation.

(iii) The crystal operator $e_{1}$ (respectively $e_{0}$ ) increases the length of the top edge on the left (resp. right) side by 1 , and otherwise does not affect that side. This implies that $\varphi_{i}$ and $\varphi_{i}^{*}$ are given by the lengths of the top and bottom edges.

Received by the editors May 9, 2012 and, in revised form, February 7, 2013.

2010 Mathematics Subject Classification. Primary 05E10; Secondary 17B67, 52B20.

The first author acknowledges support from the ANR, project ANR-09-JCJC-0102-01.

The second author acknowledges support from the ERC, project \#247049(GLC).

The third author acknowledges support from NSERC.

The fourth author acknowledges support from the NSF postdoctoral fellowship DMS-0902649. 
(iv) For a dominant weight $\Lambda$, the lowest weight crystal $B(-\Lambda) \subset B(-\infty)$ can be characterized as the set of MV polytopes such that, if the bottom vertex is placed at $-\Lambda$, the whole polytope is contained in the convex hull of the Weyl group orbit of $-\Lambda$.

In the finite type, rank $2 \mathrm{MV}$ polytopes were characterized combinatorially on a case-by-case basis in 9] using the tropical Plucker relations. In the present paper, we define MV polytopes for $\widehat{\mathrm{sl}}_{2}$ and $A_{2}^{(2)}$ using diagonals (Definition 3.4). A careful examination of the tropical Plucker relations shows that this definition also characterizes MV polytopes in the finite type case (at least for $A_{2}$ and $B_{2}$, as $G_{2}$ is still unclear). Thus, the current perspective appears to give a uniform (not case-by-case) definition of rank $2 \mathrm{MV}$ polytopes in finite and affine type.

Two recent works motivated and informed this paper. The first is the construction by the second author [5] of a conjectural realization of $B(-\infty)$ for $\widehat{\mathrm{sl}}_{2}$ using decorated lattice polytopes. Here we use new definitions for both the set of polytopes and the crystal operators, but our setup was motivated by this earlier work, and we expect that the constructions are equivalent.

The second is the construction by the other three authors 2] of MV polytopes (for symmetric finite and affine Lie algebras) associated to components of Lusztig's nilpotent varieties. We believe that the polytopes defined here essentially agree with the $\widehat{s l}_{2}$ case of that construction, and we plan to address this issue in future work. As discussed in [2], this would give a combinatorial description of MV polytopes in all symmetric affine cases 1 .

Finally, we would like to mention the work of Naito, Sagaki and Saito [17] (see also Muthiah [15]) giving a version of MV polytopes for $\widehat{\mathrm{sl}}_{n}$, so in particular, $\widehat{\mathrm{sl}}_{2}$. That construction seems to be quite different from the one given here, and it would be interesting to understand the relationship between them.

\section{BACKGROUND}

2.1. Crystals. Fix a symmetrizable Kac-Moody algebra (which for most of this paper will be $\left.\widehat{\mathrm{sl}}_{2}\right)$ and let $\Gamma=(I, E)$ be its Dynkin diagram. We are interested in the crystals $B(-\lambda)$ and $B(-\infty)$ associated with the lowest weight representation $V(-\lambda)$ and with $U^{+}(\mathfrak{g})$ respectively. These are combinatorial objects arising from the theory of crystal bases for the corresponding quantum group (see e.g. [1]). This section contains a brief explanation of the results we need, roughly following [11] and [6], to which we refer the reader for details. We begin with a combinatorial notion of crystal that includes many examples which do not arise from representations.

Definition 2.1 (see [11, Section 7.2]). A combinatorial crystal is the data $\left(B, e_{i}, f_{i}, \varepsilon_{i}, \varphi_{i}\right.$,wt) of a set $B$ along with functions wt: $B \rightarrow P$ (where $P$ is the weight lattice) and, for each $i \in I, \varepsilon_{i}, \varphi_{i}: B \rightarrow \mathbb{Z} \cup\{-\infty\}$ and $e_{i}, f_{i}: B \rightarrow B \sqcup\{\emptyset\}$, such that:

(i) $\varphi_{i}(b)=\varepsilon_{i}(b)+\left\langle\operatorname{wt}(b), \alpha_{i}^{\vee}\right\rangle$.

(ii) $e_{i}$ increases $\varphi_{i}$ by 1 , decreases $\varepsilon_{i}$ by 1 and increases wt by $\alpha_{i}$.

(iii) $f_{i} b=b^{\prime}$ if and only if $e_{i} b^{\prime}=b$.

(iv) If $\varphi_{i}(b)=-\infty$, then $e_{i} b=f_{i} b=\emptyset$.

\footnotetext{
${ }^{1}$ Since the first version of this paper was released, the relationship between our MV polytopes and the $\widehat{\mathrm{sl}}_{2}$ case of the construction from [2] has been described explicitly in [16], so the combinatorial description of MV polytopes in symmetric affine types is now complete.
} 
We often denote a combinatorial crystal by its underlying set $B$, suppressing the other data.

Definition 2.2. A morphism of combinatorial crystals is a map $\phi: B \rightarrow C$ of sets which commutes with all the structure (this is called a strict morphism in e.g. 13]).

Definition 2.3. Let $B$ and $C$ be combinatorial crystals. The tensor product $B \otimes C$ is the cartesian product $B \times C$ with crystal operators $e_{i}, f_{i}$ defined by

$f_{i}(b \otimes c)=\left\{\begin{array}{ll}f_{i}(b) \otimes c & \text { if } \varepsilon_{i}(b) \geq \varphi_{i}(c), \\ b \otimes f_{i}(c) & \text { if } \varepsilon_{i}(b)<\varphi_{i}(c),\end{array} \quad\right.$ and $\quad e_{i}(b \otimes c)= \begin{cases}e_{i}(b) \otimes c & \text { if } \varepsilon_{i}(b)>\varphi_{i}(c), \\ b \otimes e_{i}(c) & \text { if } \varepsilon_{i}(b) \leq \varphi_{i}(c) .\end{cases}$

The rest of the data is given by $\operatorname{wt}(b \otimes c)=\operatorname{wt}(b)+\operatorname{wt}(c), \varphi_{i}(b \otimes c)=\max \left\{\varphi_{i}(b), \varphi_{i}(c)\right.$ $\left.+\left\langle\operatorname{wt}(b), \alpha_{i}^{\vee}\right\rangle\right\}$, and $\varepsilon_{i}(b \otimes c)=\max \left\{\varepsilon_{i}(c), \varepsilon_{i}(b)-\left\langle\operatorname{wt}(c), \alpha_{i}^{\vee}\right\rangle\right\}$.

Definition 2.4. A lowest weight combinatorial crystal is a combinatorial crystal which has a distinguished element $b_{-}$(the lowest weight element) such that

(i) The lowest weight element $b_{-}$can be reached from any $b \in B$ by applying a sequence of $f_{i}$ for various $i \in I$.

(ii) For all $b \in B$ and all $i \in I, \varphi_{i}(b)=\max \left\{n: f_{i}^{n}(b) \neq \emptyset\right\}$.

The lowest weight element $b_{-}$of a lowest weight combinatorial crystal satisfies $\varphi_{i}\left(b_{-}\right)=0$ and $f_{i} b_{-}=\emptyset$ for all $i \in I$.

Definition 2.5 (see [11, Section 7.5]). Let $B^{(i)}$ be the crystal

$$
\ldots \stackrel{i}{\rightarrow} b^{(i)}(1) \stackrel{i}{\rightarrow} b^{(i)}(0) \stackrel{i}{\rightarrow} b^{(i)}(-1) \stackrel{i}{\rightarrow} b^{(i)}(-2) \stackrel{i}{\rightarrow} \cdots
$$

where $\operatorname{wt}\left(b^{(i)}(k)\right)=k \alpha_{i}, \varphi_{i}\left(b^{(i)}(k)\right)=k, \varepsilon_{i}\left(b^{(i)}(k)\right)=-k$, and for $j \neq i, \varphi_{j}\left(b^{(i)}(k)\right)$ $=\varepsilon_{j}\left(b^{(i)}(k)\right)=-\infty$. Here the arrows show the action of $f_{i}$.

The following is a specialization of a result of Kashiwara and Saito 13, Proposition 3.2.3].

Theorem 2.6. Let $B$ be a lowest weight combinatorial crystal. Fix an involution $*$ on $B$, assume that it fixes the lowest weight element, and define $f_{i}^{*}=* f_{i} *$ and $\varphi_{i}^{*}(b)=\varphi_{i}(* b)$. Define $\Phi_{i}: B \rightarrow B \otimes B^{(i)}$ by

$$
\Phi_{i}(b)=\left(f_{i}^{*}\right)^{\varphi_{i}^{*}(b)}(b) \otimes b^{(i)}\left(\varphi_{i}^{*}(b)\right) .
$$

If $\Phi_{i}$ is a morphism of crystals for all $i$ then $B \simeq B(-\infty)$.

It is shown in [10, Theorem 2.2.1] that there is an involution * (Kashiwara's involution) on $B(-\infty)$ such that the conditions of Theorem 2.6 hold for $(B(-\infty), *)$. Furthermore $*$ is uniquely characterized by the conditions of Theorem 2.6 , as these conditions uniquely determine the operators $f_{i}^{*}$ and hence the operators $e_{i}^{*}$, and $*$ is determined by $*\left(e_{i_{K}} \cdots e_{i_{1}} b_{-}\right)=e_{i_{K}}^{*} \cdots e_{i_{1}}^{*} b_{-}$for all $i_{1}, \ldots i_{K} \in I$. The involution * also has a simple algebraic interpretation, which can be found in [11, Section 8.3] (see also [14, Theorem 14.4.3]). The following is simply a rewording of Theorem 2.7 where we have listed the exact conditions that must be checked to see that the required maps preserve the crystal structure.

Corollary 2.7. Let $B$ be a lowest weight combinatorial crystal, of lowest weight 0 . Suppose that $e_{i} b \neq \emptyset$ for all $b \in B$ and all $i \in I$. Fix a weight-preserving involution $*$ on $B$, and define $f_{i}^{*}=* f_{i} *$ and $\varphi_{i}^{*}(b)=\varphi_{i}(* b)$. Then $B \simeq B(-\infty)$ as a crystal with involution if and only if the following three conditions hold for all $b \in B$ : 
(i) If $i \neq j$, then $f_{i}^{*} f_{j}(b)=f_{j} f_{i}^{*}(b)$. Furthermore, if $f_{i}(b), f_{i}^{*}(b) \neq \emptyset$, then $f_{i}^{*} f_{j}(b) \neq \emptyset$.

(ii) If $\varepsilon_{i}\left(\left(f_{i}^{*}\right)^{\varphi_{i}^{*}(b)}(b)\right)<\varphi_{i}^{*}(b)$, then $\varphi_{i}^{*}(b)>0$ and $f_{i}(b)=f_{i}^{*}(b)$.

(iii) If $\varepsilon_{i}\left(\left(f_{i}^{*}\right)^{\varphi_{i}^{*}(b)}(b)\right) \geq \varphi_{i}^{*}(b)$, then $f_{i}^{*} f_{i}(b)=f_{i} f_{i}^{*}(b)$, and if furthermore $f_{i} b \neq \emptyset$, then $\varphi_{i}^{*}\left(f_{i} b\right)=\varphi_{i}^{*}(b)$.

Theorem 2.8 ([11, Proposition 8.2]). Fix a dominant integral weight $\lambda=\sum_{i \in I} a_{i} \omega_{i}$, where the $\omega_{i}$ are the fundamental weights. Let $B^{\lambda}$ be the subgraph of $B(-\infty)$ consisting of those vertices $b$ such that $\varphi_{i}^{*}(b) \leq a_{i}$ for all $i \in I$. Then $f_{i}\left(B^{\lambda}\right) \subset B^{\lambda} \sqcup\{\emptyset\}$ for each $i \in I$, so one can consider the restriction $f_{i}^{\lambda}$ of $f_{i}$ to $B^{\lambda}$. Let $e_{i}^{\lambda}: B^{\lambda} \rightarrow$ $B^{\lambda} \sqcup\{\emptyset\}$ be the operator obtained from $e_{i}$ by setting $e_{i}^{\lambda}(b)=\emptyset$ if $e_{i}(b) \notin B^{\lambda}$. Let $\mathrm{wt}^{\lambda}=\mathrm{wt}-\lambda, \varepsilon_{i}^{\lambda}=\varepsilon_{i}+a_{i}$, and $\varphi_{i}^{\lambda}=\varphi_{i}$. Then $\left(B^{\lambda}, e_{i}^{\lambda}, f_{i}^{\lambda}, \varepsilon_{i}^{\lambda}, \varphi_{i}^{\lambda}, \mathrm{wt}^{\lambda}\right)$ is isomorphic to $B(-\lambda)$.

Finally, we will need the following result of Kashiwara when we discuss crystals of type $A_{2}^{(2)}$. Our statement is about $B(-\infty)$ as opposed to $B(-\lambda)$, but it follows immediately from Kashiwara's result by taking a direct limit.

Theorem 2.9 (see [12, Theorem 5.1]). Fix symmetrized Cartan matrices $N$ and $N^{\prime}$, both indexed by $I$. Assume $M=\operatorname{diag}\left\{m_{i}\right\}_{i \in I}$ is a diagonal matrix such that $N^{\prime}=M N M$. Then there is a unique embedding $S: B^{N^{\prime}}(-\infty) \rightarrow B^{N}(-\infty)$ such that:

(i) $S\left(b_{-}^{N^{\prime}}\right)=b_{-}^{N}$, where $b_{-}^{N}$ and $b_{-}^{N^{\prime}}$ are the lowest weight elements of $B^{N}(-\infty)$ and $B^{N^{\prime}}(-\infty)$, respectively.

(ii) For all $b \in B^{N^{\prime}}(-\infty)$ and each $i \in I, S\left(e_{i}(b)\right)=e_{i}^{m_{i}}(S(b))$.

2.2. The $\widehat{s l}_{2}$ root system. We refer the reader e.g. to [7] for details. The $\widehat{s l}_{2}$ root system $\Delta$ is the affine root system corresponding to the affine Dynkin diagram

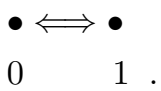

The Cartan matrix for the corresponding Kac-Moody algebra is

$$
N=\left(\begin{array}{rr}
2 & -2 \\
-2 & 2
\end{array}\right) \text {. }
$$

We denote the simple roots by $\alpha_{0}$ and $\alpha_{1}$. Define $\delta=\alpha_{0}+\alpha_{1}$.

Recall that the $\widehat{s l}_{2}$ weight space is a three-dimensional vector space containing $\alpha_{0}, \alpha_{1}$. This has a standard nondegenerate inner product $(\cdot, \cdot)$ such that

$$
\left(\alpha_{i}, \alpha_{j}\right)=\left\{\begin{array}{cc}
2 & \text { if } i=j \\
-2 & \text { if } i \neq j
\end{array}\right.
$$

Notice that $\left(\alpha_{0}, \delta\right)=\left(\alpha_{1}, \delta\right)=0$. Fix fundamental coweights $\omega_{0}, \omega_{1}$ which satisfy $\left(\alpha_{i}, \omega_{j}\right)=\delta_{i, j}$. Since this case is symmetric, these can also be taken to be the fundamental weights under the identification of weight space with coweight space.

The set of positive roots is

$$
\left\{\alpha_{0}, \alpha_{0}+\delta, \alpha_{0}+2 \delta, \ldots\right\} \sqcup\left\{\alpha_{1}, \alpha_{1}+\delta, \alpha_{1}+2 \delta, \ldots\right\} \sqcup\{\delta, 2 \delta, 3 \delta \ldots\},
$$

where the first two families consist of real roots and the third family consists of imaginary roots. All imaginary roots have multiplicity 1 . We draw these roots in the plane as 


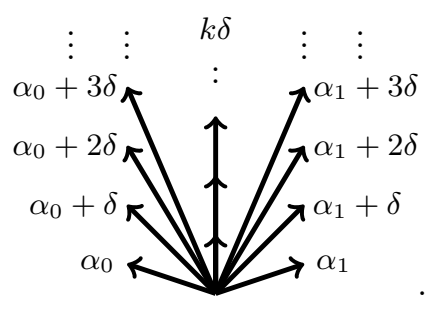

3. $\widehat{\mathrm{sl}}_{2}$ MV POLYTOPES

Here we define $\widehat{\mathrm{sl}}_{2} \mathrm{MV}$ polytopes and discuss some of their basic properties. We begin with $\widehat{\mathrm{sl}}_{2}$ GGMS polytopes, which are a more general class of decorated polytopes.

Definition 3.1. An $\widehat{s l}_{2}$ GGMS polytope is a convex polytope in $\operatorname{span}_{\mathbb{R}}\left\{\alpha_{0}, \alpha_{1}\right\}$ such that all edges are parallel to roots (see (10)). Such a polytope is called integral if all vertices lie in $\operatorname{span}_{\mathbb{Z}}\left\{\alpha_{0}, \alpha_{1}\right\}$.

We can encode a GGMS polytope by recording the position of each vertex. The vertices are labeled $\mu_{k}, \mu^{k}, \bar{\mu}_{k}, \bar{\mu}^{k}, \mu_{\infty}, \mu^{\infty}, \bar{\mu}_{\infty}, \bar{\mu}^{\infty}$ as in Figure 1 For each $k \geq 1$, define $a_{k}, a^{k}, \bar{a}_{k}, \bar{a}^{k}$ by

$$
\begin{array}{ll}
\mu_{k}-\mu_{k-1}=a_{k}\left(\alpha_{1}+(k-1) \delta\right), & \mu^{k-1}-\mu^{k}=a^{k}\left(\alpha_{0}+(k-1) \delta\right), \\
\bar{\mu}_{k}-\bar{\mu}_{k-1}=\bar{a}_{k}\left(\alpha_{0}+(k-1) \delta\right), & \bar{\mu}^{k-1}-\bar{\mu}^{k}=\bar{a}^{k}\left(\alpha_{1}+(k-1) \delta\right) .
\end{array}
$$

A polytope only has finitely many vertices, so the vertices $\mu_{k}$ must all coincide for sufficiently large $N$, as must the vertices $\mu^{k}, \bar{\mu}_{k}, \bar{\mu}^{k}$. We denote

$$
\mu_{\infty}=\lim _{k \rightarrow \infty} \mu_{k}, \mu^{\infty}=\lim _{k \rightarrow \infty} \mu^{k}, \bar{\mu}_{\infty}=\lim _{k \rightarrow \infty} \bar{\mu}_{k}, \bar{\mu}^{\infty}=\lim _{k \rightarrow \infty} \bar{\mu}^{k} .
$$

Note that $\mu^{\infty}-\mu_{\infty}$ and $\bar{\mu}^{\infty}-\bar{\mu}_{\infty}$ are both parallel to $\delta$, and that the width of the polytope is $\left(\mu_{\infty}-\bar{\mu}_{\infty}, \alpha_{1}\right) / 2$.

Definition 3.2. The diagonals of a GGMS polytope $P$ are the lines $\left(\bar{\mu}_{k}, \mu_{k-1}\right)$, $\left(\mu_{k}, \bar{\mu}_{k-1}\right),\left(\bar{\mu}^{k}, \mu^{k-1}\right)$ and $\left(\mu^{k}, \bar{\mu}^{k-1}\right)$, for $k \geq 2$.

- A diagonal $\left(\bar{\mu}_{k}, \mu_{k-1}\right)$ (respectively, $\left.\left(\mu^{k}, \bar{\mu}^{k-1}\right)\right)$ is said to be active if it is parallel to $\alpha_{0}$. In this case, the real number $s \geq 0$ such that $\bar{\mu}_{k}-\mu_{k-1}=$ $s \alpha_{0}$ (respectively, $\bar{\mu}^{k-1}-\mu^{k}=s \alpha_{0}$ ) is called the length of the diagonal.

- A diagonal $\left(\mu_{k}, \bar{\mu}_{k-1}\right)$ (respectively, $\left(\bar{\mu}^{k}, \mu^{k-1}\right)$ ) is said to be active if it is parallel to $\alpha_{1}$. In this case, the real number $s \geq 0$ such that $\mu_{k}-\bar{\mu}_{k-1}=$ $s \alpha_{0}$ (respectively, $\mu^{k-1}-\bar{\mu}^{k}=s \alpha_{1}$ ) is called the length of the diagonal.

Definition 3.3. A decorated GGMS polytope is a GGMS polytope along with a choice of two sequences $\lambda=\left(\lambda_{1} \geq \lambda_{2} \geq \cdots\right)$ and $\bar{\lambda}=\left(\bar{\lambda}_{1} \geq \bar{\lambda}_{2} \geq \cdots\right)$ of nonnegative real numbers such that $\mu^{\infty}-\mu_{\infty}=|\lambda| \delta, \bar{\mu}^{\infty}-\bar{\mu}_{\infty}=|\bar{\lambda}| \delta$, and for all sufficiently large $N, \lambda_{N}=\bar{\lambda}_{N}=0$. Here $|\lambda|=\lambda_{1}+\lambda_{2}+\cdots$ and $|\bar{\lambda}|=\bar{\lambda}_{1}+\bar{\lambda}_{2}+\cdots$. A decorated GGMS polytope is called integral if the underlying GGMS polytope is integral and all $\lambda_{k}, \bar{\lambda}_{k}$ are integers.

Definition 3.4. An $\widehat{\mathrm{sl}}_{2}$ MV polytope $P$ is a decorated $\widehat{s l}_{2}$ GGMS polytope such that

(i) For each $k \geq 2,\left(\bar{\mu}_{k}-\mu_{k-1}, \omega_{1}\right) \leq 0$ and $\left(\mu_{k}-\bar{\mu}_{k-1}, \omega_{0}\right) \leq 0$, with at least one of these being an equality. 


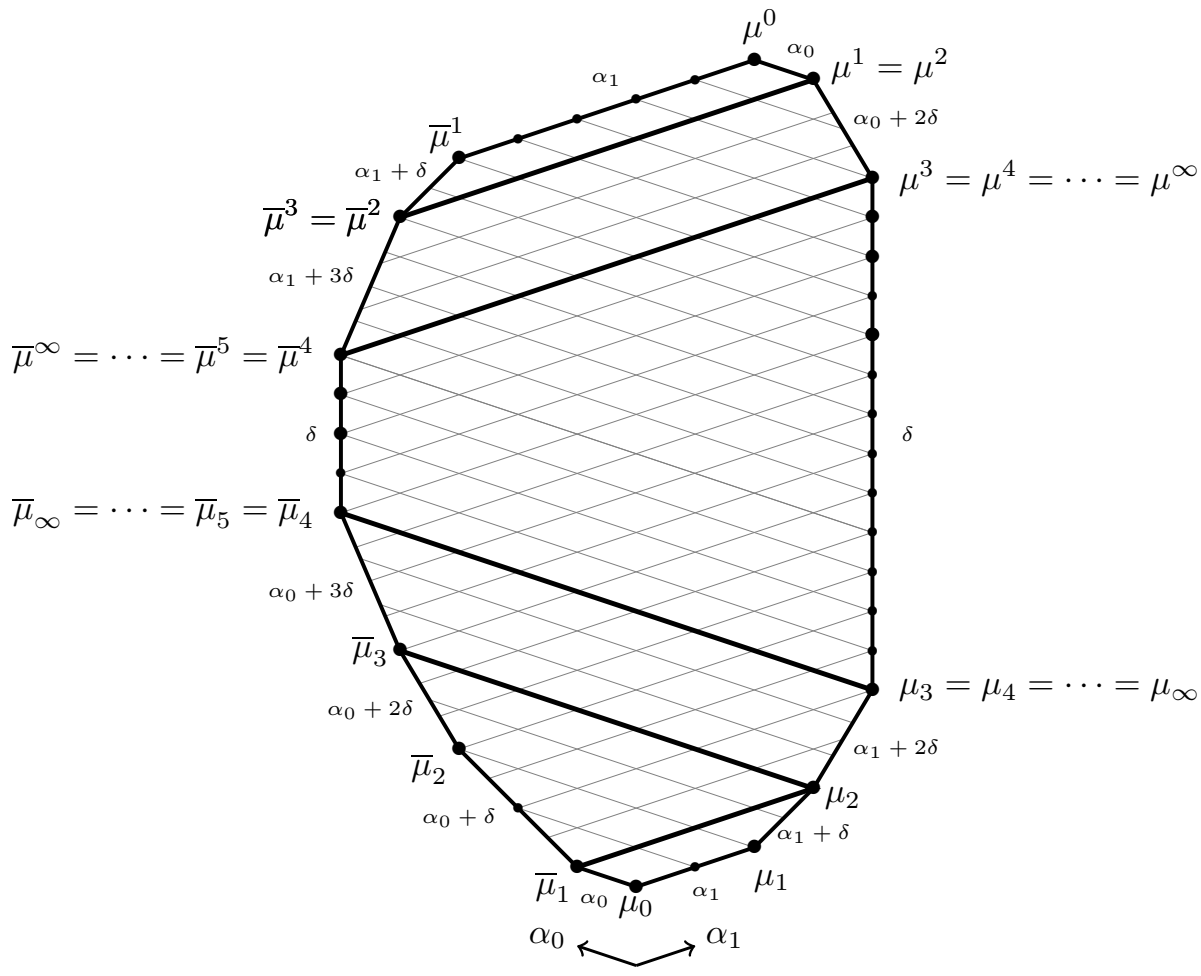

Figure 1. An integral $\widehat{\mathrm{sl}}_{2}$ MV polytope $P$. The partitions labeling the vertical edges are indicated by including extra vertices on the vertical edges, such that the edge is cut into the pieces indicated by the partition. Here $a_{1}=2, a_{2}=1, a_{3}=1, \lambda_{1}=9, \lambda_{2}=2$, $\lambda_{3}=1, \lambda_{4}=1, a^{3}=1, a^{1}=1, \bar{a}_{1}=1, \bar{a}_{2}=2, \bar{a}_{3}=1, \bar{a}_{4}=1$, $\bar{\lambda}_{1}=2, \bar{\lambda}_{2}=1, \bar{\lambda}_{3}=1, \bar{a}^{4}=1, \bar{a}^{2}=1, \bar{a}^{1}=5$, and all other $a_{k}, a^{k}, \bar{a}_{k}, \bar{a}^{k}, \lambda_{k}, \bar{\lambda}_{k}$ are 0 . The bold diagonals show a system of diagonals $S$ such that $P$ is of type $S$ (since $\left(\bar{\mu}_{2}, \mu_{1}\right)$ is also active there is a second system of diagonals $S^{\prime}$ such that $P$ is also of type $\left.S^{\prime}\right)$. All the quadrilaterals obtained by cutting the polytope along the diagonals in $S$ are themselves MV polytopes.

(ii) For each $k \geq 2,\left(\bar{\mu}^{k}-\mu^{k-1}, \omega_{0}\right) \geq 0$ and $\left(\mu^{k}-\bar{\mu}^{k-1}, \omega_{1}\right) \geq 0$, with at least one of these being an equality.

(iii) If $\left(\mu_{\infty}, \bar{\mu}_{\infty}\right)$ and $\left(\mu^{\infty}, \bar{\mu}^{\infty}\right)$ are parallel then $\lambda=\bar{\lambda}$. Otherwise, one is obtained from the other by removing a part of size $\left(\mu_{\infty}-\bar{\mu}_{\infty}, \alpha_{1}\right) / 2$ (i.e., the width of the polytope).

(iv) $\lambda_{1}, \bar{\lambda}_{1} \leq\left(\mu_{\infty}-\bar{\mu}_{\infty}, \alpha_{1}\right) / 2$.

We denote by $\mathcal{M V}$ the set of integral $\widehat{\mathrm{sl}}_{2} \mathrm{MV}$ polytopes up to translation (i.e., $P_{1}=P_{2}$ in $\mathcal{M V}$ if $P_{1}=P_{2}+\mu$ for some weight $\mu$ ).

Condition (ii) implies that for any MV polytope $P$ and each $k \geq 2$, at least one of the diagonals $\left(\mu_{k}, \bar{\mu}_{k-1}\right)$ and $\left(\bar{\mu}_{k}, \mu_{k-1}\right)$ is active. Consequently, $\left(\bar{\mu}_{\infty}, \mu_{\infty}\right)$ is parallel to $\alpha_{0}$ or to $\alpha_{1}$. Likewise, condition (iii) implies at least one of the diagonals 
$\left(\mu^{k}, \bar{\mu}^{k-1}\right)$ and $\left(\bar{\mu}^{k}, \mu^{k-1}\right)$ is active. Consequently, $\left(\bar{\mu}^{\infty}, \mu^{\infty}\right)$ is parallel to $\alpha_{0}$ or to $\alpha_{1}$.

Remark 3.5. It is immediate from Definition 3.4 that the set $\mathcal{M V}$ is preserved under vertical reflection (i.e., the linear map defined by $\alpha_{1} \mapsto-\alpha_{0}$ and $\alpha_{0} \mapsto-\alpha_{1}$ ), horizontal reflection (i.e., the linear map $\alpha_{0} \leftrightarrow \alpha_{1}$ ) and negation (which is just the composition of the first two maps).

Let $P$ be an MV polytope. If we cut $P$ along any active diagonal, then both halves are themselves MV polytopes, as we see from Definition 3.4. Further, cutting $P$ along a maximal system of non-intersecting active diagonals, we can decompose $P$ into a union of quadrilaterals, each of which is itself an MV polytope. Up to symmetry, only four types of quadrilaterals can occur, which are shown in Figure 2 ,

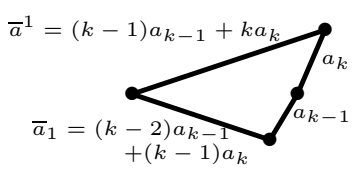

(1)

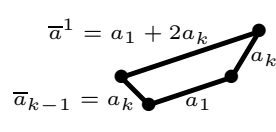

(2)

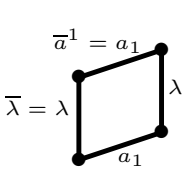

(3)

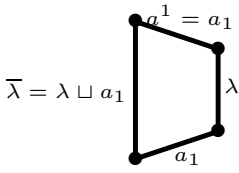

(4)

Figure 2. Any MV polytope can be cut along active diagonals into quadrilaterals which are themselves MV polytopes. The possible quadrilaterals that can arise are highly restricted by the fact that they have no non-degenerate active diagonals. In fact, the only ones that can occur are those shown here, along with those obtained from them by symmetries (reflection in one of the axes or negation). Here $k \geq 2, \lambda$ is a partition with $\lambda_{1} \leq a_{1}$, and in (2) we require $a_{1} \geq(k-2) a_{k}$.

In finite type, MV polytopes are defined by conditions known as the tropical Plücker relations, which involve minimums. By specifying for each relation which term should be the minimum, one obtains different types of MV polytopes (see [9. Section 3.4]). In our situation there are also many types of MV polytopes, depending on which diagonals are active.

To formalize this, we introduce formal symbols $\tau_{k}, \tau^{k}$ for $k \geq 0$ and $\bar{\tau}_{k}, \bar{\tau}^{k}$ for $k \geq 1$. These represent the labels for the vertices of an abstract, general MV polytope. The diagonals of this polytope would be the pairs $\left(\bar{\tau}_{k}, \tau_{k-1}\right),\left(\tau_{k}, \bar{\tau}_{k-1}\right)$, $\left(\bar{\tau}^{k}, \tau^{k-1}\right)$ and $\left(\tau^{k}, \bar{\tau}^{k-1}\right)$, for $k \geq 2$.

Definition 3.6. A system of diagonals is a subset $S$ of

$$
\left\{\left(\bar{\tau}_{k}, \tau_{k-1}\right),\left(\tau_{k}, \bar{\tau}_{k-1}\right),\left(\bar{\tau}^{k}, \tau^{k-1}\right),\left(\tau^{k}, \bar{\tau}^{k-1}\right): k \geq 2\right\}
$$

which

- contains exactly one of $\left\{\left(\bar{\tau}_{k}, \tau_{k-1}\right),\left(\tau_{k}, \bar{\tau}_{k-1}\right)\right\}$ and exactly one of $\left\{\left(\bar{\tau}^{k}, \tau^{k-1}\right),\left(\tau^{k}, \bar{\tau}^{k-1}\right)\right\}$ for each $k \geq 2$,

- either contains $\left(\bar{\tau}_{k}, \tau_{k-1}\right)$ for all sufficiently large $k$ or contains $\left(\tau_{k}, \bar{\tau}_{k-1}\right)$ for all sufficiently large $k$, and

- either contains $\left(\bar{\tau}^{k}, \tau^{k-1}\right)$ for all sufficiently large $k$ or contains $\left(\tau^{k}, \bar{\tau}^{k-1}\right)$ for all sufficiently large $k$. 
Definition 3.7. Fix a system of diagonals $S$. We say a GGMS polytope $P$ is of type $S$ if

- the diagonal $\left(\bar{\mu}^{k}, \mu^{k-1}\right)$ is active whenever $\left(\bar{\tau}^{k}, \tau^{k-1}\right) \in S$,

- the diagonal $\left(\mu^{k}, \bar{\mu}^{k-1}\right)$ is active whenever $\left(\tau^{k}, \bar{\tau}^{k-1}\right) \in S$,

- the diagonal $\left(\bar{\mu}_{k}, \mu_{k-1}\right)$ is active whenever $\left(\bar{\tau}_{k}, \tau_{k-1}\right) \in S$,

- the diagonal $\left(\mu_{k}, \bar{\mu}_{k-1}\right)$ is active whenever $\left(\tau_{k}, \bar{\tau}_{k-1}\right) \in S$.

It is immediate from Definition 3.4 that every MV polytope is of type $S$ for some system of diagonals $S$. The following essentially shows that there are nondegenerate MV polytopes of type $S$ for every system of diagonals $S$.

Proposition 3.8. For any system of diagonals $S$, there is some $P \in \mathcal{M V}$ of type $S$ which is not of type $S^{\prime}$ for any other system of diagonals $S^{\prime}$.

Proof. We say that a pair $\left(\bar{\tau}_{k}, \tau_{k-1}\right)$ or $\left(\tau^{k}, \bar{\tau}^{k-1}\right)$ is an $\alpha_{0}$-diagonal, and a pair $\left(\tau_{k}, \bar{\tau}_{k-1}\right)$ or $\left(\bar{\tau}^{k}, \tau^{k-1}\right)$ is an $\alpha_{1}$-diagonal.

We proceed by induction on the number of times the type of diagonal ( $\alpha_{0}$ versus $\left.\alpha_{1}\right)$ in $S$ changes as you move from the bottom to the top. We will prove inductively that the desired $P$ exists and can be chosen such that all the active diagonals below coincide the first time the type of diagonal changes. If the number of changes is 0 , one can take $P$ to be a line segment parallel to $\alpha_{0}$ or $\alpha_{1}$.

Assume the number of changes is at least 1 , and without loss of generality assume $\left(\tau_{2}, \bar{\tau}_{1}\right) \in S$. Let $\Phi$ be the set of $\alpha_{1}$-diagonals in $S$ which are below all $\alpha_{0}$-diagonals in $S$, and let $S^{\prime}$ be the system of diagonals obtained by replacing all diagonals in $\Phi$ with the corresponding $\alpha_{0}$-diagonals. By induction, we can find the required polytope $P^{\prime}$ for $S^{\prime}$.

There are three cases, based on whether $\Phi$ is

(4) of the form $\left\{\left(\tau_{j}, \bar{\tau}_{j-1}\right): j<k\right\}$ for some $k$,

(5) of the form $\left\{\left(\tau_{j}, \bar{\tau}_{j-1}\right): j \geq 2\right\}$, or

(6) of the form $\left\{\left(\tau_{j}, \bar{\tau}_{j-1}\right): j \geq 2\right\} \cup\left\{\left(\bar{\tau}^{j}, \tau^{j-1}\right): j>k\right\} \quad$ for some $k$.

In each case, one can glue a quadrilateral or a triangle at the bottom of $P^{\prime}$ to obtain the desired $P$, as shown in Figure 3. This can be done such that all the edges have rational length, and then we can rescale to get an element of $\mathcal{M V}$ satisfying the required conditions.

Definition 3.9. A Lusztig datum is a choice of a tuple $\mathbf{a}=\left(a_{k}, \lambda_{k}, a^{k}\right)_{k \in \mathbb{N}}$ of non-negative real numbers such that

(i) for all sufficiently large $k, a_{k}=a^{k}=\lambda_{k}=0$, and

(ii) $\lambda_{1} \geq \lambda_{2} \geq \cdots$.

Definition 3.10. The right Lusztig data of a decorated GGMS polytope is the data $\mathbf{a}=\left(a_{k}, \lambda_{k}, a^{k}\right)_{k \in \mathbb{N}}$. The left Lusztig data is $\overline{\mathbf{a}}=\left(\bar{a}_{k}, \bar{\lambda}_{k}, \bar{a}^{k}\right)_{k \in \mathbb{N}}$.

Theorem 3.11. For each Lusztig datum a, there is a unique $\widehat{\mathrm{sl}}_{2} M V$ polytope $P_{\mathbf{a}}$ whose right Lusztig data is given by $\mathbf{a}$. Furthermore $P_{\mathbf{a}}$ is integral if and only if all $a_{k}, \lambda_{k}$ and $a^{k}$ are integers.

Remark 3.12. We often use the notation $\overline{\mathbf{a}}$ to denote the left Lusztig data of the polytope $P_{\mathbf{a}}$ specified by Theorem 3.11 . 

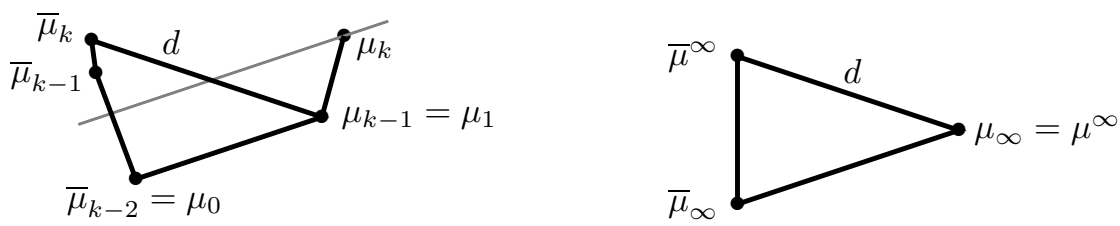

Figure 3. The inductive step for the proof of Proposition 3.8, In the left diagram, $\Phi$ is as in (4) for the value of $k$ shown. Inductively we can find a polytope $P^{\prime}$ for $S^{\prime}$, and since $\left(\bar{\mu}_{k-1}, \mu_{k}\right)$ is not active in $P^{\prime}, \bar{\mu}_{k}$ is strictly above the line through $\mu_{k}$ parallel to $\alpha_{1}$. Thus we can append the shown quadrilateral, where $\bar{\mu}_{k-1}$ is chosen to be sufficiently close to $\bar{\mu}_{k}$, but not equal to $\bar{\mu}_{k}$. In the right diagram, $\Phi$ is as in (5), and we can append a triangle to $S^{\prime}$, where the partition associated to the vertical edge is a single part. The case when $\Phi$ is as in (6) is similar to the left diagram.

Remark 3.13. In finite type, the lengths of the edges along one side of an MV polytope (or, in rank greater than two, along certain paths from the bottom to the top vertex) is the data used by Lusztig to parameterize the corresponding element in $B(-\infty)$. Lusztig's construction uses a longest element of the Weyl group, so does not generalize immediately to $\widehat{\mathrm{sl}}_{2}$. However, 4] (see also [3]) gives an analogue of Lusztig parameterization for $\widehat{s l}_{2}$. It would be interesting to determine if our combinatorics can be related to this algebraic construction 2

3.1. Proof of Theorem 3.11. Our proof is effective: we present a recursive algorithm to construct the polytope $P_{\mathbf{a}}$ by reducing to cases $\mathbf{a}^{\prime}$ where the integer

(7) $h(\mathbf{a}):=2\left|\left\{k \geq 2: a_{k} \neq 0\right\}\right|+2\left|\left\{k \geq 2: a^{k} \neq 0\right\}\right|+\left(1\right.$ if $\left.a_{1} \neq 0\right)+\left(1\right.$ if $\left.a^{1} \neq 0\right)$

has been reduced. We begin by considering some simple Lusztig data which will serve as building blocks.

Lemma 3.14. Theorem 3.11 holds for a Lusztig data of the form $\left(0, \ldots, 0, a_{k}, a_{k+1}\right.$, $0, \ldots, 0)$, and the corresponding left Lusztig data is $\left(\bar{a}_{1}, 0, \ldots, 0, \bar{a}^{1}\right)$, with $\bar{a}_{1}=$ $(k-1) a_{k}+k a_{k+1}$ and $\bar{a}^{1}=k a_{k}+(k+1) a_{k+1}$.

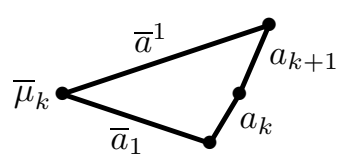

Lemma 3.15. Theorem 3.11 holds for a Lusztig data of the form $\left(a_{1}, 0, \ldots, 0, a^{1}\right)$. Moreover, for the MV polytope with this right Lusztig data:

(i) The left Lusztig data is given as follows:

- If $a_{1}=a^{1}$, then $\bar{a}_{k}=\bar{a}^{k}=0$ for all $k$ and $\bar{\lambda}=\left(a_{1} \geq 0 \geq 0 \geq \ldots\right)$ (left picture below).

- If $a_{1}<a^{1}$, then there is an integer $r \geq 1$ such that $\frac{r-1}{r} \leq \frac{a_{1}}{a^{1}} \leq \frac{r}{r+1}$ and

$$
\left(\begin{array}{c}
\bar{a}_{r} \\
\bar{a}_{r+1}
\end{array}\right)=\left(\begin{array}{cc}
r & -(r+1) \\
-(r-1) & r
\end{array}\right)\left(\begin{array}{l}
a^{1} \\
a_{1}
\end{array}\right) ;
$$

\footnotetext{
${ }^{2}$ Since the first version of this paper was released, this has been done. See [16].
} 
all the other $\bar{a}_{k}$ are zero, as are all the $\bar{a}^{k}$ and all the $\bar{\lambda}_{k}$ (right picture below).

- The case $a_{1}>a^{1}$ can be obtained from the previous one by vertical reflection.
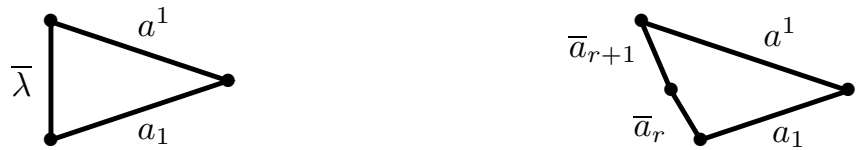

(ii) Let $k \geq 2$. In order that $\mu^{0}=\bar{\mu}_{k}$, it is necessary and sufficient that $k a_{1} \leq(k-1) a^{1}$, and then $\bar{a}_{k}=\max \left(0,(k-1) a_{1}-(k-2) a^{1}\right)$. In order that $\mu_{0}=\bar{\mu}_{k-2}$, it is necessary and sufficient that $(k-2) a^{1} \leq(k-1) a_{1}$, and then $\bar{a}_{k-1}=\max \left(0,(k-1) a^{1}-k a_{1}\right)$.

Lemma 3.16. Theorem 3.11 holds for a Lusztig data of the form $\left(a_{1}, 0, \ldots, 0, a_{k}\right.$, $0, \ldots, 0)$. If $a_{1} \geq(k-2) a_{k}$, then the left Lusztig data is $\left(0, \ldots, 0, \bar{a}_{k-1}, 0, \ldots, 0, \bar{a}^{1}\right)$, with $\bar{a}_{k-1}=a_{k}$ and $\bar{a}^{1}=a_{1}+2 a_{k}$ (left picture below). If $a_{1} \leq(k-2) a_{k}$, then $\bar{a}^{1}=k a_{k}$ and the $M V$ polytope is obtained by stacking a triangle above the $M V$ polytope with right Lusztig data $\left(a_{1}, 0, \ldots, 0,(k-1) a_{k}\right)$ (right picture below). In any case, $\bar{a}^{1}=\max \left(a_{1}+2 a_{k}, k a_{k}\right)$.
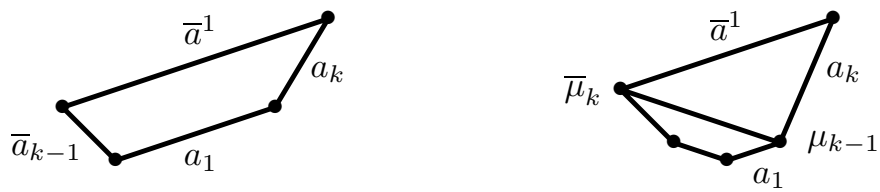

Lemma 3.17. Theorem 3.11 holds for a Lusztig datum of the form $\left(0, \ldots, 0, a_{k}\right.$, $\left.0, \ldots, 0, a^{1}\right)$, where $k \geq 2$. If $a^{1} \geq(k+1) a_{k}$, then the left Lusztig data is $\left(\bar{a}_{1}, 0, \ldots, 0, \bar{a}_{k+1}, 0, \ldots, 0\right)$, with $\bar{a}_{1}=a^{1}-2 a_{k}$ and $\bar{a}_{k+1}=a_{k}$ (left picture below). If $a^{1} \leq(k+1) a_{k}$, then $\bar{a}_{1}=(k-1) a_{k}$ and the $M V$ polytope is obtained by stacking the $M V$ polytope with right Lusztig data $\left(k a_{k}, 0, \ldots, 0, a^{1}\right)$ above a triangle (right picture below). In any case, $\bar{a}_{1}=\max \left(a^{1}-2 a_{k},(k-1) a_{k}\right)$.
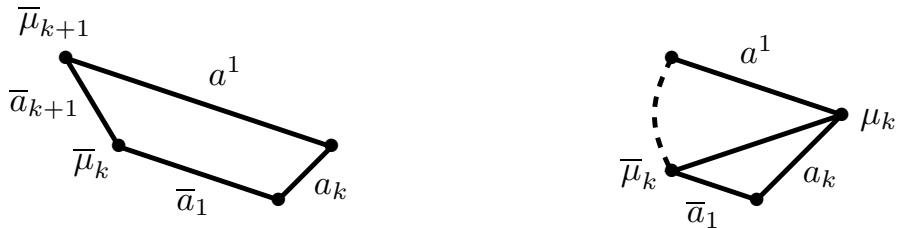

Lemma 3.18. Theorem 3.11 holds for a Lusztig datum of the form $\left(a_{1}, 0, \ldots, 0, \lambda\right.$, $\left.0, \ldots, 0, a^{1}\right)$.

Proof of Lemmas 3.14, 3.15, 3.16, 3.17, and 3.18. These are all proven by elementary arguments. As an example, we show Lemma 3.15. We also provide some details for Lemma 3.18, since this requires a little more care than the others.

Proof for Lemma 3.15: The existence of an MV polytope with the prescribed right Lusztig data follows from the explicit description given in the statement of the lemma. It remains to show the uniqueness.

To this aim, consider an MV polytope with right Lusztig data $\left(a_{1}, 0, \ldots, 0, a^{1}\right)$. We first notice that $\mu_{1}=\mu_{\infty}=\mu^{\infty}=\mu^{1}$, so if $\left(\bar{\mu}^{\infty}, \mu^{\infty}\right)$ is parallel to $\alpha_{0}$, then 
$\bar{\mu}^{\infty}=\mu^{0}$, and if $\left(\mu^{\infty}, \bar{\mu}^{\infty}\right)$ is parallel to $\alpha_{1}$, then $\bar{\mu}^{\infty}=\mu_{0}$. So $\bar{\mu}^{\infty} \in\left\{\mu^{0}, \mu_{0}\right\}$, and likewise $\bar{\mu}_{\infty} \in\left\{\mu^{0}, \mu_{0}\right\}$.

Consider the case $a_{1}=a^{1}$. Then $\mu^{0}-\mu_{0}=a_{1} \delta$. If we had $\bar{\mu}^{\infty} \neq \mu^{0}$, then $\mu^{0}-\bar{\mu}^{\infty}$ would be a nonzero nonnegative linear combination of roots $\alpha_{1}+k \delta$, hence would be of the form $x \alpha_{1}+y \delta$ with $x>0$. Then $\bar{\mu}^{\infty}-\mu_{0}$ would be equal to $\left(a_{1}-y\right) \delta-x \alpha_{1}$, and so $\bar{\mu}^{\infty} \notin\left\{\mu^{0}, \mu_{0}\right\}$, a contradiction. Therefore $\bar{\mu}^{\infty}=\mu^{0}$. Likewise, $\bar{\mu}_{\infty}=\mu_{0}$. Condition (iii) in Definition 3.4 then gives the announced result.

Consider now the case $a_{1}<a^{1}$. This time, $\bar{\mu}^{\infty}=\bar{\mu}_{\infty}=\mu^{0}$. If some vertex $\bar{\mu}_{r}$ for $r \geq 2$ is different from both $\mu_{0}$ and $\mu^{0}$, then neither $\left(\bar{\mu}_{r}, \mu_{r-1}\right)$ nor $\left(\mu_{r+1}, \bar{\mu}_{r}\right)$ is active, so $\left(\mu_{r}, \bar{\mu}_{r-1}\right)$ and $\left(\bar{\mu}_{r+1}, \mu_{r}\right)$ are active, which forces $\bar{\mu}_{r-1}=\mu_{0}$ and $\bar{\mu}_{r+1}=\mu^{0}$, and by a similar argument the same conclusion holds when $r=1$. A straightforward calculation concludes the proof of (ii). The statement (iii) is an immediate corollary of (i).

Sketch of proof for Lemma 3.18: Here we need to distinguish between four cases.

- If $a_{1}=a^{1} \geq \lambda_{1}$, then $\bar{\mu}_{\infty}=\mu_{0}$ and $\bar{\mu}^{\infty}=\mu^{0}$, and therefore the polytope $P_{\mathbf{a}}$ is a trapezoid with left decoration $\bar{\lambda}=\left(a_{1} \geq \lambda_{1} \geq \lambda_{2} \geq \cdots\right)$ (left picture below).

- If $\lambda_{1}>\max \left(a_{1}, a^{1}\right)$, then $\left(\bar{\mu}_{\infty}, \mu_{\infty}\right)$ is parallel to $\alpha_{0}$ and $\left(\mu^{\infty}, \bar{\mu}^{\infty}\right)$ is parallel to $\alpha_{1}$, and the polytope $P_{\mathbf{a}}$ is obtained by stacking the MV polytope with right Lusztig data $\left(a_{1}, 0, \ldots, 0, \lambda_{1}\right)$, a trapezoid with decoration $\bar{\lambda}=\left(\lambda_{2} \geq \lambda_{3} \geq \cdots\right)$, and the MV polytope with right Lusztig data $\left(\lambda_{1}, 0, \ldots, 0, a^{1}\right)$ (central picture below).

- If $a^{1}>a_{1}$ and $a^{1} \geq \lambda_{1}$, then $\bar{\mu}^{\infty}=\mu^{0},\left(\bar{\mu}_{\infty}, \mu_{\infty}\right)$ is parallel to $\alpha_{0}$, and the polytope $P_{\mathbf{a}}$ is obtained by stacking the MV polytope with right Lusztig data $\left(a_{1}, 0, \ldots, 0, a^{1}\right)$ and the parallelogram with left decoration $\lambda$ (right picture below).

- The case $a_{1}>a^{1}$ and $a_{1} \geq \lambda_{1}$ can be obtained from the previous one by vertical reflection.
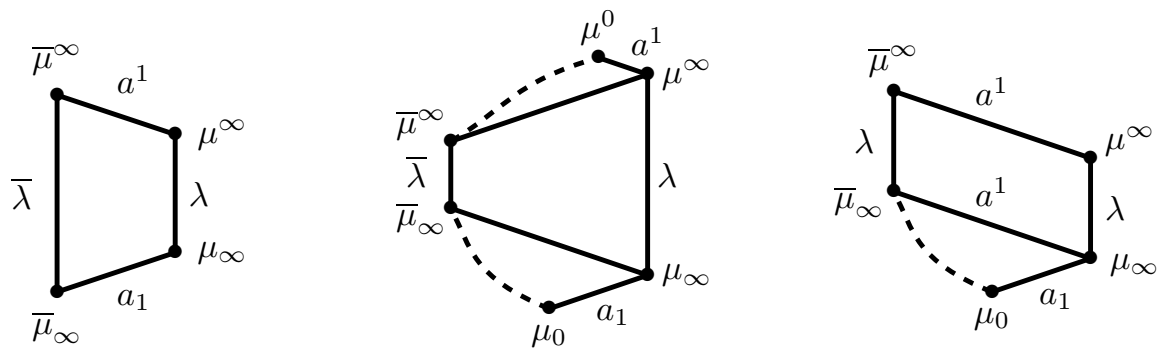

In the general case, the existence and uniqueness of $P_{\mathbf{a}}$ will be established alongside the following properties.

Proposition 3.19. Let $\mathbf{a}$ be a Lusztig datum such that $a_{1}>0$ and set $\mathbf{b}=$ $\left(0, a_{2}, a_{3}, \ldots, a^{1}\right)$. Let $k \geq 2$ be such that $a_{2}=\cdots=a_{k-1}=0$. Then $(k-2) \bar{b}_{1} \leq$ $(k-1) a_{1}$ if and only if $\bar{a}_{1}=\cdots=\bar{a}_{k-2}=0$, and if these assertions hold true, then $\bar{a}_{k-1}=\max \left(a_{k},(k-1) \bar{b}_{1}-k a_{1}\right)$. 
Proposition 3.20. Let $\mathbf{a}$ be a Lusztig datum and let $k \geq 2$ such that $a_{1}=\cdots=$ $a_{k-1}=0 . \quad$ Set $\mathbf{d}=\left(0, \ldots, 0, a_{k+1}, a_{k+2}, \ldots, a^{1}\right)$. Then $\bar{a}_{1}=\max \left(\bar{d}_{1}-2 a_{k}\right.$, $\left.(k-1) a_{k}+k a_{k+1}\right)$ and $\bar{a}_{2}=\cdots=\bar{a}_{k}=0$.

Proof of Theorem 3.11, Proposition 3.19 and Proposition 3.20. We prove these simultaneously by induction on $h(\mathbf{a})$ from (7), the case where $h(\mathbf{a})=0$ being handled by Lemma 3.18. The inductive step breaks into three cases, each of which requires several arguments.

Induction step when $a_{1}>0$ and there exists $\ell \geq 2$ such that $a_{\ell}>0$ :

Denote by $k$ the smallest $\ell \geq 2$ such that $a_{\ell}>0$. Set $\mathbf{b}=\left(0, \ldots, 0, a_{k}, a_{k+1}, \ldots, a^{1}\right)$ and $\mathbf{d}=\left(0, \ldots, 0, a_{k+1}, a_{k+2}, \ldots, a^{1}\right)$. Then $h(\mathbf{b})=h(\mathbf{a})-1$ and $h(\mathbf{d})=h(\mathbf{a})-3$, so by induction, we know that $P_{\mathbf{b}}$ and $P_{\mathbf{d}}$ exist and are unique, and that $\bar{b}_{1}=$ $\max \left(\bar{d}_{1}-2 a_{k},(k-1) a_{k}+k a_{k+1}\right)$ and $\bar{b}_{2}=\cdots=\bar{b}_{k}=0$, where $\overline{\mathbf{b}}$ and $\overline{\mathbf{d}}$ are the left Lusztig data of $P_{\mathbf{b}}$ and $P_{\mathbf{d}}$ (Proposition 3.20).

Set also $\mathbf{c}=\left(c_{1}, 0, \ldots, 0, a_{k+1}, a_{k+2}, \ldots, a^{1}\right)$, where $c_{1}=\max \left(a_{1}+2 a_{k}, k a_{k}\right)$. Since $h(\mathbf{c})=h(\mathbf{a})-1$, by induction again, we know that $P_{\mathbf{c}}$ exists, is unique, and satisfies Proposition 3.19.

By definition, at least one of the diagonals $\left(\bar{\mu}_{k}, \mu_{k-1}\right)$ or $\left(\mu_{k}, \bar{\mu}_{k-1}\right)$ is active in any MV polytope with right Lusztig data a, which gives two possibilities:
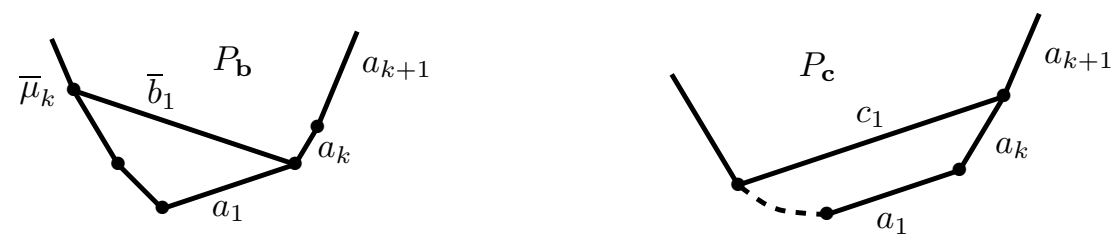

Uniqueness in Theorem 3.11: Assume $Q$ is an MV polytope with right Lusztig data a.

- If $\left(\bar{\mu}_{k}, \mu_{k-1}\right)$ is active in $Q$, then we can cut $Q$ in two along this diagonal (left picture). The polytope above the diagonal is necessarily $P_{\mathbf{b}}$, and so below the diagonal, we have the MV polytope with right Lusztig data $\left(a_{1}, 0, \ldots, 0, \bar{b}_{1}\right)$, which is also fully determined (Lemma 3.15$)$. Thus $Q$ is fully determined. Since $Q$ is MV, in the bottom polytope, the right upper edge must coincide with the diagonal $\left(\bar{\mu}_{k}, \mu_{k-1}\right)$, so, by Lemma 3.15 (iii), $k a_{1} \leq(k-1) \bar{b}_{1}$ and $\bar{a}_{k}=\max \left(0,(k-1) a_{1}-(k-2) \bar{b}_{1}\right)$. Since

$$
\bar{\mu}_{k-1}=\mu_{1}+\bar{b}_{1} \alpha_{0}-\bar{a}_{k}\left(\alpha_{0}+(k-1) \delta\right) \quad \text { and } \quad \mu_{k}=\mu_{1}+a_{k}\left(\alpha_{1}+(k-1) \delta\right) \text {, }
$$

the condition $\left(\omega_{0}, \mu_{k}-\bar{\mu}_{k-1}\right) \leq 0$ gives $(k-1) a_{k}-\bar{b}_{1}+k \bar{a}_{k} \leq 0$, so that

$$
\bar{b}_{1} \geq(k-1) a_{k}+k\left((k-1) a_{1}-(k-2) \bar{b}_{1}\right),
$$

which rearranges to $(k-1) \bar{b}_{1} \geq k a_{1}+a_{k}$.

- If $\left(\bar{\mu}_{k}, \mu_{k-1}\right)$ is inactive in $Q$, then $\left(\mu_{k}, \bar{\mu}_{k-1}\right)$ is active, and we can cut $Q$ along this latter diagonal (right picture). The polytope below this diagonal is necessarily the polytope with right Lusztig data $\left(a_{1}, 0, \ldots, 0, a_{k}, 0, \ldots\right)$, which is fully determined by Lemma 3.16. The polytope above the diagonal has necessarily right Lusztig data $\mathbf{c}$, so is $P_{\mathbf{c}}$. Thus $Q$ is fully determined. Since we assume $Q$ is MV and $\left(\bar{\mu}_{k}, \mu_{k-1}\right)$ is inactive, we have $\left(\omega_{1}, \bar{\mu}_{k}-\right.$ $\left.\mu_{k-1}\right)<0$. Since $\bar{\mu}_{k}=\mu_{k}-c_{1} \alpha_{1}+\bar{c}_{k}\left(\alpha_{0}+(k-1) \delta\right)$ and $\mu_{k-1}=\mu_{k}-$ 
$a_{k}\left(\alpha_{1}+(k-1) \delta\right)$, this gives $k a_{k}+(k-1) \bar{c}_{k}<c_{1}$. In particular, we see that $c_{1}>k a_{k}$, and so by the definition of $\mathbf{c}$ we have $c_{1}=a_{1}+2 a_{k}$.

Now the bottom vertex of $P_{\mathbf{c}}$ is its vertex $\bar{\mu}_{k-1}$, so $\bar{c}_{1}=\cdots=\bar{c}_{k-1}=0$. By Proposition 3.19 applied to $\mathbf{c}$, we have $\bar{c}_{k}=\max \left(c_{k+1}, k \bar{d}_{1}-(k+1) c_{1}\right)$. It is then easy to rewrite the inequality $k a_{k}+(k-1) \bar{c}_{k}<c_{1}$ as the system $(k-1) \bar{d}_{1}<k a_{1}+(2 k-1) a_{k} \quad$ and $\quad(k-2) a_{k}+(k-1) a_{k+1}<a_{1}$.

Remembering $\bar{b}_{1}=\max \left(\bar{d}_{1}-2 a_{k},(k-1) a_{k}+k a_{k+1}\right)$, we obtain $(k-1) \bar{b}_{1}<$ $k a_{1}+a_{k}$.

It follows that there is always at most one $Q \in \mathcal{M} V$ with right Lusztig data a; its diagonal $\left(\bar{\mu}_{k}, \mu_{k-1}\right)$ must be active if $(k-1) \bar{b}_{1} \geq k a_{1}+a_{k}$ and inactive if $(k-1) \bar{b}_{1}<k a_{1}+a_{k}$.

\section{Existence in Theorem 3.11:}

- Assume first that $(k-1) \bar{b}_{1} \geq k a_{1}+a_{k}$. Then $(k-1) \bar{b}_{1} \geq k a_{1}$, so the polytope with right Lusztig datum $\left(a_{1}, 0, \ldots, 0, \bar{b}_{1}\right)$ is a quadrilateral, whose upper vertex is $\bar{\mu}_{k}$ (Lemma 3.15 (iii)). Since $\bar{b}_{2}=\cdots=\bar{b}_{k}=0$, we can place the polytope $P_{\mathbf{b}}$ above this quadrilateral and obtain a GGMS polytope. By Lemma 3.15 (iii), we have $\bar{a}_{k}=\max \left(0,(k-1) a_{1}-(k-2) \bar{b}_{1}\right)$; combining $\bar{b}_{1} \geq(k-1) a_{k}$ with $(k-1) \bar{b}_{1} \geq k a_{1}+a_{k}$, we get $\bar{b}_{1}-(k-1) a_{k} \geq k \bar{a}_{k}$. As in the uniqueness part of the proof, we then compute $\left(\omega_{0}, \mu_{k}-\bar{\mu}_{k-1}\right)=$ $(k-1) a_{k}-\bar{b}_{1}+k \bar{a}_{k}$. This is nonpositive, hence our candidate polytope is MV.

- Assume now that $(k-1) \bar{b}_{1} \leq k a_{1}+a_{k}$. Then

$$
(k-1) \bar{d}_{1} \leq k a_{1}+(2 k-1) a_{k} \leq k\left(a_{1}+2 a_{k}\right) \leq k c_{1} .
$$

Proposition 3.19 applied to $\mathbf{c}$ then guarantees that $\bar{c}_{1}=\cdots=\bar{c}_{k-1}=0$ and that $\bar{c}_{k}=\max \left(c_{k+1}, k \bar{d}_{1}-(k+1) c_{1}\right)$. In particular, the bottom vertex of $P_{\mathbf{c}}$ is $\bar{\mu}_{k-1}$. Moreover,

$$
(k-1)\left((k-1) a_{k}+k a_{k+1}\right) \leq(k-1) \bar{b}_{1} \leq k a_{1}+a_{k}
$$

gives $(k-1)^{2} a_{k} \leq k a_{1}+a_{k}$, and then $a_{1} \geq(k-2) a_{k}$. Therefore by Lemma 3.16 the MV polytope with right Lusztig data $\left(a_{1}, 0, \ldots, 0, a_{k}, 0, \ldots\right)$ can be placed below $P_{\mathbf{c}}$ to obtain a GGMS polytope.

The inequality $a_{1} \geq(k-2) a_{k}$ gives $c_{1}=a_{1}+2 a_{k}$. From $(k-1) \bar{d}_{1} \leq$ $k a_{1}+(2 k-1) a_{k}$ and $(k-1)\left((k-1) a_{k}+k a_{k+1}\right) \leq k a_{1}+a_{k}$, we deduce $k a_{k}+(k-1) \bar{c}_{k}<c_{1}$. It follows that $\left(\omega_{0}, \bar{\mu}_{k}-\mu_{k-1}\right) \leq 0$, which implies that our candidate polytope is MV.

\section{Proposition 3.19:}

Let $\ell \geq 2$ be such that $a_{2}=\cdots=a_{\ell-1}=0$. Since $a_{k}>0$, we necessarily have $\ell \leq k$.

- Assume first that $(k-1) \bar{b}_{1} \geq k a_{1}+a_{k}$. Then $P_{\mathbf{a}}$ is given by the left diagram in equation (8). The numbers $\bar{a}_{1}, \ldots, \bar{a}_{\ell}$ for $P_{\mathbf{a}}$ come from the quadrilateral at the bottom. Applying Lemma 3.15 (iii) to this quadrilateral, we see that $\bar{a}_{1}=\cdots=\bar{a}_{\ell-2}=0$ if and only if $(\ell-2) \bar{b}_{1} \leq(\ell-1) a_{1}$, and if these assertions hold true, then $\bar{a}_{\ell-1}=\max \left(0,(\ell-1) \bar{b}_{1}-\ell a_{1}\right)$.

To complete the proof of Proposition 3.19, it now suffices to check that $\max \left(0,(\ell-1) \bar{b}_{1}-\ell a_{1}\right)=\max \left(a_{\ell},(\ell-1) \bar{b}_{1}-\ell a_{1}\right)$. This equality is shown 
by looking separately at the cases $\ell<k$ and $\ell=k$. In the first case, one simply notices that $a_{\ell}=0$. In the second case, one uses the assumption $(k-1) \bar{b}_{1} \geq k a_{1}+a_{k}$.

- Assume now that $(k-1) \bar{b}_{1} \leq k a_{1}+a_{k}$. Then $P_{\mathbf{a}}$ is given by the right diagram in equation (8), so we have $\bar{a}_{1}=\cdots=\bar{a}_{k-2}=0$ and $\bar{a}_{k-1}=a_{k}$. Furthermore, we saw in the existence proof above that $a_{1} \geq(k-2) a_{k}$, which implies

$$
(k-1) \bar{b}_{1} \leq k a_{1}+\frac{a_{1}}{k-2}=\frac{(k-1)^{2}}{k-2} a_{1},
$$

whence $\bar{b}_{1} / a_{1} \leq(k-1) /(k-2) \leq(\ell-1) /(\ell-2)$. Therefore both assertions $\bar{a}_{1}=\cdots=\bar{a}_{\ell-2}=0$ and $(\ell-2) \bar{b}_{1} \leq(\ell-1) a_{1}$ hold true.

It remains to check that $\bar{a}_{\ell-1}=\max \left(a_{\ell},(\ell-1) \bar{b}_{1}-\ell a_{1}\right)$. If $\ell \leq k-1$, this comes from the fact that $\bar{a}_{\ell-1}=a_{\ell}=0$ and that $\bar{b}_{1} / a_{1} \leq(k-1) /(k-2) \leq$ $\ell /(\ell-1)$. If $\ell=k$, this comes from the equality $\bar{a}_{k-1}=a_{k}$ and from the assumption $(k-1) \bar{b}_{1} \leq k a_{1}+a_{k}$.

Induction step when $a_{1}=0$ and $a_{\ell}>0$ for some $\ell \geq 2$ :

Denote by $k$ the smallest value such that $a_{k} \neq 0$. Set $\mathbf{b}=\left(0, \ldots, 0, a_{k+1}, a_{k+2}\right.$, $\left.\ldots, a^{1}\right)$ and $\mathbf{d}=\left(0, \ldots, 0, a_{k+2}, a_{k+3}, \ldots, a^{1}\right)$. Then $h(\mathbf{b})=h(\mathbf{a})-2$ and $h(\mathbf{d}) \leq$ $h(\mathbf{a})-2$, so by induction, $P_{\mathbf{b}}$ and $P_{\mathbf{d}}$ exist and are unique, and we have $\bar{b}_{1}=$ $\max \left(\bar{d}_{1}-2 a_{k+1}, k a_{k+1}+(k+1) a_{k+2}\right)$ and $\bar{b}_{2}=\cdots=\bar{b}_{k+1}=0$, where $\overline{\mathbf{b}}$ and $\overline{\mathbf{d}}$ are the left Lusztig data of $P_{\mathbf{b}}$ and $P_{\mathbf{d}}$ (Proposition 3.20).

Set also $\mathbf{c}=\left(c_{1}, 0, \ldots, 0, a_{k+2}, a_{k+3}, \ldots, a^{1}\right)$, where $c_{1}=k a_{k}+(k+1) a_{k+1}$. Since $h(\mathbf{c}) \leq h(\mathbf{a})-1$, by induction, we know that $P_{\mathbf{c}}$ exists, is unique, and satisfies Proposition 3.19 .

By definition, at least one of the diagonals $\left(\mu_{k+1}, \bar{\mu}_{k}\right)$ or $\left(\bar{\mu}_{k+1}, \mu_{k}\right)$ is active in any MV polytope with right Lusztig data a, giving two possibilities:
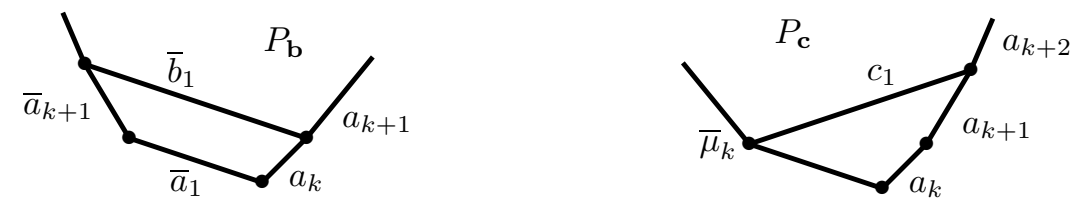

Uniqueness in Theorem 3.11: Assume $Q \in \mathcal{M} V$ has right Lusztig data a.

- If $\left(\mu_{k+1}, \bar{\mu}_{k}\right)$ is active in $Q$, then we can cut $Q$ in two along this diagonal (right picture). The polytope below is necessarily the polytope with right Lusztig data $\left(0, \ldots, 0, a_{k}, a_{k+1}, 0, \ldots, 0\right)$, which is fully determined by Lemma 3.14. Its left upper edge has length $c_{1}$, and thus the MV polytope above the diagonal is necessarily $P_{\mathbf{c}}$. Thus $Q$ is fully determined. Since $P_{\mathbf{c}}$ is obtained as the part of $Q$ above the diagonal $\left(\mu_{k+1}, \bar{\mu}_{k}\right)$, the lower vertex of $P_{\mathbf{c}}$ is its vertex $\bar{\mu}_{k}$, so $\bar{c}_{1}=\cdots=\bar{c}_{k}=0$. Hence by Proposition 3.19 applied to $P_{\mathbf{c}}$, we have $k \bar{d}_{1} \leq(k+1) c_{1}$ and $\bar{c}_{k+1}=\max \left(c_{k+2},(k+1) \bar{d}_{1}-(k+2) c_{1}\right)$. Since

$$
\bar{\mu}_{k+1}=\bar{\mu}_{k}+\bar{c}_{k+1}\left(\alpha_{0}+k \delta\right) \text { and } \mu_{k}=\bar{\mu}_{k}+c_{1} \alpha_{1}-a_{k+1}\left(\alpha_{1}+k \delta\right) \text {, }
$$

the equation $\left(\omega_{1}, \bar{\mu}_{k+1}-\mu_{k}\right) \leq 0$ gives $\bar{c}_{k+1} \leq a_{k}$. This translates to the system

$$
a_{k+2} \leq a_{k} \quad \text { and } \quad \bar{d}_{1} \leq(k+1) a_{k}+(k+2) a_{k+1} .
$$


Remembering that $\bar{b}_{1}=\max \left(\bar{d}_{1}-2 a_{k+1}, k a_{k+1}+(k+1) a_{k+2}\right)$, we conclude that $\bar{b}_{1} \leq(k+1) a_{k}+k a_{k+1}$.

- If $\left(\mu_{k+1}, \bar{\mu}_{k}\right)$ is inactive in $Q$, then $\left(\bar{\mu}_{k+1}, \mu_{k}\right)$ is active, and we can cut $Q$ along this latter (left picture). The polytope above the diagonal is necessarily $P_{\mathbf{b}}$, and so below the diagonal we have the MV polytope with right Lusztig data $\left(0, \ldots, 0, a_{k}, 0, \ldots, 0, \bar{b}_{1}\right)$. The latter is fully determined by Lemma 3.17. Thus $Q$ is fully determined, and we even know that $\bar{a}_{1}=$ $\max \left(\bar{b}_{1}-2 a_{k},(k-1) a_{k}\right)$. Now $\left(\mu_{k+1}, \bar{\mu}_{k}\right)$ is inactive, so $\left(\omega_{0}, \mu_{k+1}-\bar{\mu}_{k}\right)<0$. Since $\bar{\mu}_{k}=\bar{a}_{1} \alpha_{0}$ and $\mu_{k+1}=a_{k}\left(\alpha_{1}+(k-1) \delta\right)+a_{k+1}\left(\alpha_{1}+k \delta\right)$, this gives $\bar{a}_{1}>(k-1) a_{k}+k a_{k+1}$, and therefore $\bar{b}_{1}>(k+1) a_{k}+k a_{k+1}$.

It follows that there can only ever be at most one $Q \in \mathcal{M} V$ with right Lusztig data a, where the diagonal $\left(\mu_{k+1}, \bar{\mu}_{k}\right)$ must be active if $\bar{b}_{1} \leq(k+1) a_{k}+k a_{k+1}$, and inactive if $\bar{b}_{1}>(k+1) a_{k}+k a_{k+1}$.

\section{Existence in Theorem 3.11;}

- Assume first that $\bar{b}_{1} \geq(k+1) a_{k}+k a_{k+1}$. Then a fortiori $\bar{b}_{1} \geq(k+$ 1) $a_{k}$, and so, by Lemma 3.17, the MV polytope with right Lusztig data $\left(0, \ldots, 0, a_{k}, 0, \ldots, 0, \bar{b}_{1}\right)$ is the quadrilateral at the bottom on the left diagram in (9). For this polytope, the vertex $\bar{\mu}_{k+1}$ coincides with the top vertex and we have $\bar{a}_{k+1}=a_{k}$. Remembering that $\bar{b}_{2}=\cdots=\bar{b}_{k+1}=0$, we see that we can place $P_{\mathbf{b}}$ above this quadrilateral and get a GGMS polytope. A direct computation then gives $\left(\omega_{0}, \mu_{k+1}-\bar{\mu}_{k}\right)=k a_{k+1}-\bar{b}_{1}+(k+1) \bar{a}_{k+1}$. This is nonpositive, hence our candidate polytope is MV.

- Assume now that $\bar{b}_{1} \leq(k+1) a_{k}+k a_{k+1}$. Then $\bar{d}_{1} \leq(k+1) a_{k}+(k+2) a_{k+1}$ and $a_{k+2} \leq a_{k}$, and therefore

$$
k \bar{d}_{1} \leq k(k+1) a_{k}+k(k+2) a_{k+1} \leq(k+1) c_{1},
$$

where the last inequality comes because $c_{1}=k a_{k}+(k+1) a_{k+1}$. Proposition 3.19 applied to $\mathbf{c}$ leads to $\bar{c}_{1}=\cdots=\bar{c}_{k}=0$ and $\bar{c}_{k+1}=\max \left(c_{k+2},(k+\right.$ 1) $\left.\bar{d}_{1}-(k+2) c_{1}\right)$. Therefore, the bottom vertex of $P_{\mathbf{c}}$ is its vertex $\bar{\mu}_{k}$, so $P_{\mathbf{c}}$ can be stacked above the MV polytope with right Lusztig data $\left(0, \ldots, 0, a_{k}, a_{k+1}, 0, \ldots, 0\right)$, as shown on the right picture in (9). Furthermore, since

$$
(k+1) \bar{d}_{1}-(k+2) c_{1} \leq(k+1)\left[(k+1) a_{k}+(k+2) a_{k+1}\right]-(k+2) c_{1}=a_{k},
$$

we get $\bar{c}_{k+1} \leq a_{k}$. This shows that $\left(\omega_{1}, \bar{\mu}_{k+1}-\mu_{k}\right)=k \bar{c}_{k+1}-c_{1}+(k+1) a_{k+1}$ is nonpositive, which implies that our candidate polytope is MV.

\section{Proposition 3.20:}

This is immediate from the explicit construction of $P_{\mathbf{a}}$ given above and from Lemmas 3.14 and 3.17 .

\section{Induction step where $a_{k}=0$ for all $k \geq 2$ :}

\section{Theorem 3.11:}

If $a^{k}=0$ for all $k \geq 2$, then Theorem 3.11 for a follows from Lemma 3.18 (this case should really be thought of as the initialization of the induction).

Suppose now that there is $k \geq 2$ such that $a^{k}>0$, and consider the Lusztig data $\mathbf{a}^{\prime}=\left(a^{1}, a^{2}, \ldots, \lambda, \ldots, a_{2}, a_{1}\right)$. Applying one of our induction steps above to $\mathbf{a}^{\prime}$, we see that Theorem 3.11 holds for $\mathbf{a}^{\prime} .\left(h\left(\mathbf{a}^{\prime}\right)=h(\mathbf{a})\right.$, so this trick does not drive us 
backwards in the induction.) The image of $P_{\mathbf{a}^{\prime}}$ by vertical reflection is then the unique MV polytope $P_{\mathbf{a}}$.

\section{Proposition 3.19:}

Our assumption here is that $a_{1}>0$ and $a_{\ell}=0$ for all $\ell \geq 2$, which means that $\mu_{1}=\mu_{\infty}$. Let $\mathbf{b}$ be as in the statement of the proposition; by induction, the left Lusztig data of $P_{\mathbf{b}}$ satisfies $\bar{b}_{2}=\bar{b}_{3}=\cdots=0$ (Proposition [3.20). Let $k \geq 2$. If $\bar{b}_{1} \geq a_{1}$, then by Lemma 3.15 we can glue $P_{\mathbf{b}}$ and the polytope with right Lusztig data $\left(a_{1}, 0, \ldots, 0, \bar{b}_{1}\right)$ to get an MV polytope, and the statement follows from Lemma 3.15 (iii). Otherwise, $\left(\bar{\mu}_{\infty}, \mu_{\infty}\right)$ cannot be parallel to $\alpha_{0}$, so must be parallel to $\alpha_{1}$. Then all the $\bar{a}_{k}$ are in fact 0 , and again the statement holds.

\section{Proposition 3.20:}

We are here in the situation where $a_{k}=0$ for all $k \geq 1$, whence $\mu_{0}=\mu_{\infty}$. Then $\left(\bar{\mu}_{\infty}, \mu_{\infty}\right)$ is parallel to $\alpha_{0}$ in $P_{\mathbf{a}}$ and $\bar{\mu}_{\infty}=\bar{\mu}_{1}$, which implies that $\bar{a}_{k}=0$ for all $k \geq 2$. The result then follows from the observation that $\mathbf{d}=\mathbf{a}$.

These three cases cover all possibilities, so the induction is complete.

Proposition 3.21. For any nontrivial $P \in \mathcal{M V}$, either $a_{1}$ or $\bar{a}_{1}$ is nonzero, and either $a^{1}$ or $\bar{a}^{1}$ is nonzero.

Proof. Let a be a Lusztig data such that $a_{1}=\bar{a}_{1}=0$. By Proposition 3.20, we see that all $a_{k}$ are zero. The same argument applied after horizontal reflection gives that all $\bar{a}_{k}$ are zero. In the MV polytope $P_{\mathbf{a}}$, we thus have $\mu_{0}=\mu_{\infty}=\bar{\mu}_{\infty}$. By Definition 3.4 (iv), we get $\lambda=\bar{\lambda}=0$. We thus have $\mu^{\infty}=\bar{\mu}^{\infty}$. Obvious weight considerations then show that all $a^{k}$ and all $\bar{a}^{k}$ are zero. Thus a is trivial.

Remark 3.22. The induction used to prove Theorem 3.11 can be viewed as an algorithm that explicitly constructs $P_{\mathbf{a}}$ and $\overline{\mathbf{a}}$ from $\mathbf{a}$. Following this algorithm step by step, one can check that

$$
\begin{gathered}
\bar{a}_{1}=\max \left\{(k-1) a_{k}+(k-2) a_{k-1}-2 a_{k-2}-\cdots-2 a_{2}-2 a_{1}, \text { for } k \geq 2,\right. \\
\lambda_{1}-\cdots-2 a_{3}-2 a_{2}-2 a_{1}, k a^{k}+(k+1) a^{k+1}+2 a^{k+2}+2 a^{k+3}+\cdots \\
\left.-2 a_{k-2}-\cdots-2 a_{2}-2 a_{1}, \text { for } k \geq 1\right\} .
\end{gathered}
$$

\section{Crystal structure}

Definition 4.1. Fix $P \in \mathcal{M} V$ with right and left Lusztig data a and $\overline{\mathbf{a}}$ respectively. Then $e_{0}(P)$ is the MV polytope with right Lusztig data $e_{0}(\mathbf{a})$ and $e_{1}(P)$ is the MV polytope with left Lusztig datum $e_{1}(\overline{\mathbf{a}})$, where $e_{0}(\mathbf{a})$ agrees with a except that $e_{0}(\mathbf{a})^{1}=a^{1}+1$, and $e_{1}(\overline{\mathbf{a}})$ agrees with $\overline{\mathbf{a}}$ except that $e_{1}(\overline{\mathbf{a}})^{1}=\bar{a}^{1}+1$.

Similarly, $f_{0}(P)$ is the MV polytope with right data $f_{0}(\mathbf{a})$ and $f_{1}(P)$ is the MV polytope with left Lusztig data $f_{1}(\overline{\mathbf{a}})$, where $f_{0}(\mathbf{a})$ agrees with a except that $f_{0}(\mathbf{a})^{1}=a^{1}-1$ and $f_{1}(\overline{\mathbf{a}})$ agrees with $\overline{\mathbf{a}}$ except that $f_{1}(\overline{\mathbf{a}})^{1}=\bar{a}^{1}-1$, and if $a^{1}$ or $\bar{a}^{1}$ are zero, then $f_{0}$ or $f_{1}$ sends that polytope to $\emptyset$.

Definition 4.2. Fix $P \in \mathcal{M} V$ with right Lusztig data a and left Lusztig data $\overline{\mathbf{a}}$.

(i) $\operatorname{wt}(P)=\mu^{0}-\mu_{0}$.

(ii) $\varphi_{0}(P)=a^{1}, \varphi_{1}(P)=\bar{a}^{1}$.

(iii) $\varepsilon_{0}(P)=\varphi_{0}(P)-\left(\operatorname{wt}(P), \alpha_{0}\right)$ and $\varepsilon_{1}(P)=\varphi_{1}(P)-\left(\operatorname{wt}(P), \alpha_{1}\right)$. 
Definition 4.3. The involution $*$ on $\mathcal{M} V$ negates the polytope. Recalling that elements of $\mathcal{M V}$ are only defined up to translation, this can be described algebraically as follows: for Lusztig data

$$
\mathbf{a}=\left(a_{1}, a_{2}, \ldots, \lambda, \ldots, a^{2}, a^{1}\right),
$$

$P_{\mathbf{a}}^{*}$ has left Lusztig data $\mathbf{b}=\left(\bar{b}_{k}, \nu_{k}, \bar{b}^{k}\right)$, where $\bar{b}_{k}=a^{k}, \nu_{k}=\lambda_{k}, \bar{b}^{k}=a_{k}$.

Define operators $e_{0}^{*}, e_{1}^{*}, f_{0}^{*}, f_{1}^{*}$ on $B(-\infty)$ by $e_{0}^{*}=* \circ e_{0} \circ *, e_{1}^{*}=* \circ e_{1} \circ *, f_{0}^{*}=$ $* \circ f_{0} \circ *, f_{1}^{*}=* \circ f_{1} \circ *$, and similarly $\varepsilon_{0}^{*}=\varepsilon_{0} \circ *, \quad \varepsilon_{1}^{*}=\varepsilon_{1} \circ *, \varphi_{0}^{*}=\varphi_{0} \circ *, \quad \varphi_{1}^{*}=$ $\varphi_{1} \circ *$.

Remark 4.4. The $*$ operators can also be defined as in Definition 4.1 but where $\bar{a}_{1}$ and $a_{1}$ get modified instead of $a^{1}$ and $\bar{a}^{1}$.

Theorem 4.5. $\left(\mathcal{M} V, e_{0}, e_{1}, f_{0}, f_{1}, \varepsilon, \varphi, \mathrm{wt}\right)$ is isomorphic to the crystal $B(-\infty)$. Furthermore, * is Kashiwara's involution.

The proof of Theorem 4.5 will be delayed until Section 4.1 We first discuss an immediate consequence which allows us to see the crystals $B(-\Lambda)$ of all integrable lowest weight representations using our MV polytopes. For a dominant integral weight $\Lambda=c_{0} \Lambda_{0}+c_{1} \Lambda_{1}$ for $\widehat{s l}_{2}$, define $\mathcal{M} V^{\Lambda}$ to be the subset of $\mathcal{M} V$ consisting of those MV polytopes $P_{\mathbf{a}}$ such that $a_{1} \leq c_{1}$ and $\bar{a}_{1} \leq c_{0}$. The following is immediate from Theorems 2.8 and 4.5 .

Corollary 4.6. Fix a dominant integral weight $\Lambda=c_{0} \Lambda_{0}+c_{1} \Lambda_{1}$ for $\widehat{\mathrm{sl}}_{2}$. Let $e_{i}^{\Lambda}, f_{i}^{\Lambda}$ be the operators inherited from the operators on $\mathcal{M V}$ by setting all $P \notin \mathcal{M} V^{\Lambda}$ equal to $\emptyset$. Let $\mathrm{wt}^{\Lambda}=\mathrm{wt}-\Lambda, \varepsilon_{i}^{\Lambda}=\varepsilon_{i}+c_{i}$ and $\varphi_{i}^{\Lambda}=\varphi_{i}$. Then $\left(\mathcal{M} V^{\Lambda}, e_{i}^{\Lambda}, f_{i}^{\Lambda}, \varphi_{i}^{\Lambda}, \varepsilon_{i}^{\Lambda}, \mathrm{wt}^{\Lambda}\right)$ is isomorphic to $B(-\Lambda)$.

Recall that MV polytopes are only defined up to translation by weights. For a dominant weight $\Lambda$ and an MV polytope $P$, let $P^{\Lambda}$ denote the representative for $P$ whose lowest vertex $\mu_{0}$ equals $-\Lambda$.

Theorem 4.7. For any dominant weight $\Lambda$, we have

$$
\mathcal{M} V^{\Lambda}=\left\{P \in \mathcal{M} V: P^{\Lambda} \subset \operatorname{Conv} W(-\Lambda)\right\},
$$

where $W$ denotes the Weyl group of $\widehat{\mathrm{sl}}_{2}$ and Conv denotes convex hull.

Proof. Write $\Lambda=c_{0} \Lambda_{0}+c_{1} \Lambda_{1}$. If $P^{\Lambda} \subset \operatorname{Conv} W(-\Lambda)$, then the containment of the vertices $\mu_{1}$ and $\bar{\mu}_{1}$ forces $a_{1} \leq c_{1}$ and $\bar{a}_{1} \leq c_{0}$.

We now turn to the converse implication. Set

$$
v_{k}=s_{1} s_{0} s_{1} \cdots s_{i}(-\Lambda) \text { and } \bar{v}_{k}=s_{0} s_{1} s_{0} \cdots s_{i}(-\Lambda),
$$

where in all cases there are exactly $k$ reflections. These are the vertices of Conv $W(-\Lambda)$. Pick $P \in \mathcal{M} V^{\Lambda}$, and let $\mu_{k}, \bar{\mu}_{k}, \mu^{k}, \bar{\mu}^{k}$ be the vertices of $P^{\Lambda}$. We first show that, for each $k$,

$$
\begin{aligned}
& v_{k}-\mu_{k} \in \operatorname{span}_{\mathbb{R}_{\geq 0}}\left(\alpha_{1}, \alpha_{1}+(k-1) \delta\right) \quad \text { and } \\
& \bar{v}_{k}-\bar{\mu}_{k} \in \operatorname{span}_{\mathbb{R}_{\geq 0}}\left(\alpha_{0}, \alpha_{0}+(k-1) \delta\right) .
\end{aligned}
$$


We do this by induction on $k$, as illustrated in Figure 4 and explained below:

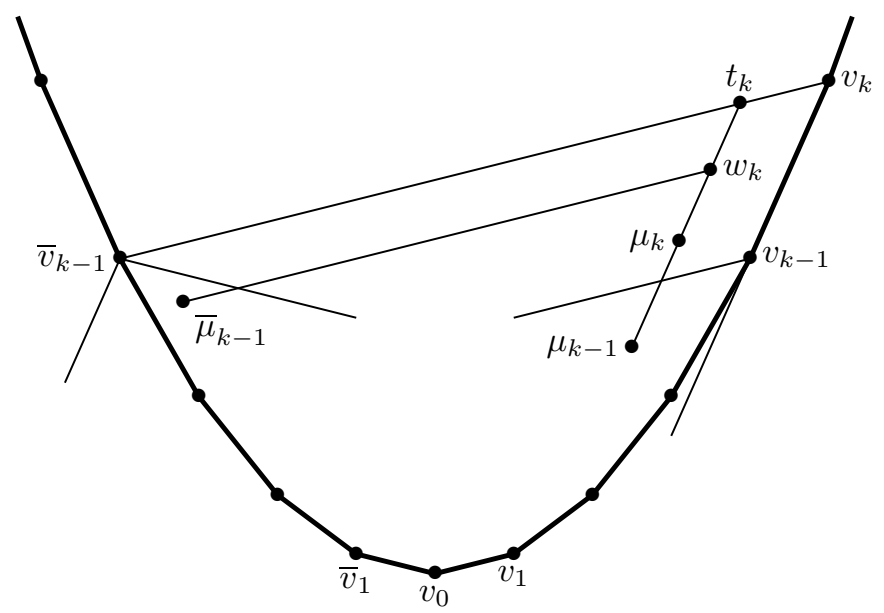

FiguRE 4. Diagram accompanying the proof of Theorem 4.7

Let $w_{k}$ be the intersection of the line through $\bar{\mu}_{k-1}$ parallel to $\alpha_{1}$ and the line through $\mu_{k-1}$ parallel to $\alpha_{1}+(k-1) \delta$. By the definition of an MV polytope, $w_{k}-\mu_{k}=t\left(\alpha_{1}+(k-1) \delta\right)$ for some $t \geq 0$.

By induction, we can write

$v_{k-1}-\mu_{k-1}=x \alpha_{1}+y\left(\alpha_{1}+(k-1) \delta\right) \quad$ and $\quad \bar{v}_{k-1}-\bar{\mu}_{k-1}=\bar{x}\left(-\alpha_{1}\right)+\bar{y}\left(\alpha_{1}+(k-1) \delta\right)$ for $x, y, \bar{x}, \bar{y} \geq 0$ (since the positive cone spanned by $\left\{\alpha_{1}, \alpha_{1}+(k-1) \delta\right\}$ contains that spanned by $\left\{\alpha_{1}, \alpha_{1}+(k-2) \delta\right\}$, and the positive cone spanned by $\left\{-\alpha_{1}, \alpha_{1}+(k-1) \delta\right\}$ contains that spanned by $\left.\left\{\alpha_{0}, \alpha_{0}+(k-2) \delta\right\}\right)$. It follows that

$$
v_{k}-w_{k}=x \alpha_{1}+\bar{y}\left(\alpha_{1}+(k-1) \delta\right),
$$

and hence

$$
v_{k}-\mu_{k}=x \alpha_{1}+(\bar{y}+t)\left(\alpha_{1}+(k-1) \delta\right) .
$$

Notice that $x$ and $\bar{y}+t$ are both nonnegative. Similarly, $\bar{v}_{k}-\bar{\mu}_{k} \in \operatorname{span}_{\mathbb{R}_{>0}}\left(\alpha_{0}, \alpha_{0}+\right.$ $(k-1) \delta)$, completing the proof of (10).

We are now ready to finish the proof of the theorem. We must show that all the vertices $\mu_{k}, \bar{\mu}_{k}, \mu^{k}, \bar{\mu}^{k}$ belong to $\operatorname{Conv} W(-\Lambda)$. We first consider the vertices $\mu_{k}$. We proceed by induction on $k$, the case $k=0$ being trivial. So assume that, for some $k, \mu_{k-1} \in \operatorname{Conv} W(-\Lambda)$. Let $t_{k}$ be the intersection of the line through $v_{k}$ parallel to $\alpha_{1}$ and the line through $\mu_{k}$ parallel to $\alpha_{1}+(k-1) \delta$. By (10), we know that $v_{k}-t_{k}$ is a nonnegative multiple of $\alpha_{1}$ and $t_{k}-\mu_{k}$ is a nonnegative multiple of $\alpha_{1}+(k-1) \delta$. The affine cone

$$
\bar{v}_{k-1}-\left[\mathbb{R}_{>0} \alpha_{1}+\mathbb{R}_{\geq 0}\left(\alpha_{1}+(k-1) \delta\right)\right]
$$

is disjoint from Conv $W(-\Lambda)$, so cannot contain $\mu_{k-1}$, which forces $t_{k}-\bar{v}_{k-1}$ to be a nonnegative multiples of $\alpha_{1}$. Therefore $t_{k}$ belongs to the line segment with endpoints $\bar{v}_{k-1}$ and $v_{k}$. Likewise, both $t_{k}-\mu_{k}$ and $\mu_{k}-\mu_{k-1}$ are nonnegative multiples of $\alpha_{1}+(k-1) \delta$, and so $\mu_{k}$ belongs to the line segment with endpoints 
$t_{k}$ and $\mu_{k-1}$. Therefore $\mu_{k}$ lies in the convex hull of $\left\{\bar{v}_{k-1}, v_{k}, \mu_{k-1}\right\}$, and hence in Conv $W(-\Lambda)$.

A similar argument to the above shows that $\bar{\mu}_{k}$ lies in $\operatorname{Conv} W(-\Lambda)$. That the $\mu^{k}, \bar{\mu}^{k}$ are in the convex hull then follows form the well-known fact that Conv $W(-\Lambda)$ is stable by translation by $t \delta$ for any $t \geq 0$, which for completeness we now prove: Let $\alpha$ in the classical root lattice and let $t_{ \pm \alpha} \in W$ be the translation by $\pm \alpha$. Any element $\lambda \in W(-\Lambda)$ has the same level as $-\Lambda$, namely $-\left(c_{0}+c_{1}\right)$. Using [7, (6.5.2)], we see that the midpoint of $t_{\alpha} \lambda$ and $t_{-\alpha} \lambda$ is $\lambda+\frac{c_{0}+c_{1}}{2}|\alpha|^{2} \delta$. Choosing $\alpha$ large enough, we deduce that $\lambda+t \delta$ belongs to the convex hull of $W \lambda$ for any $t \geq 0$.

4.1. Proof of Theorem 4.5. Before beginning, let us give an outline of the proof. We first show that $\mathcal{M V}$ is a lowest weight combinatorial crystal. It remains to check the three conditions of Corollary 2.7. Condition (ii) is straightforward. To handle (iii) and (iii), we first give a very explicit description of the operators $f_{i}$. If $P$ does not have an active diagonal parallel to $\alpha_{1}$ then this description will show that $\varphi_{i}^{*}(b)>0$ and $f_{0}(P)=f_{0}^{*}(P)$. If $P \in \mathcal{M} V$ does have an active diagonal parallel to $\alpha_{1}$, then our description will show that $f_{0}^{*} f_{0}(P)=f_{0} f_{0}^{*}(P)$ and $\varphi_{0}^{*}\left(f_{0} b\right)=\varphi_{0}^{*}(b)$. The final step will be to show that the condition $\varepsilon_{0}\left(\left(f_{0}^{*}\right)^{\varphi_{0}^{*}(P)}(P)\right) \geq \varphi_{0}^{*}(P)$ is equivalent to the condition that $P$ has an active diagonal parallel to $\alpha_{1}$. The argument with the roles of 0 and 1 reversed is completely symmetric.

Lemma 4.8. $\left(\mathcal{M V}, e_{i}, f_{i}, \varepsilon, \varphi, \mathrm{wt}\right)$ is a lowest weight combinatorial crystal.

Proof. One readily checks that $\left(\mathcal{M} V, e_{i}, f_{i}, \varepsilon, \varphi\right.$, wt $)$ is a combinatorial crystal that enjoys condition (ii) in Definition 2.4. We prove condition (i) by induction on the weight. The lowest weight element is the trivial polytope (i.e., a single point, up to translation). Let $P$ be a nontrivial integral MV polytope. By Proposition 3.21, either $a^{1} \neq 0$ or $\bar{a}^{1} \neq 0$. Then by definition $f_{i}(P) \neq \emptyset$ for $i=0$ or $i=1$. Since $f_{i}(P)$ has smaller weight than $P$, by our induction hypothesis, the trivial polytope can be reached by applying a sequence of lowering operators $f_{j}$ to $f_{i}(P)$. Adding $f_{i}$ to this sequence, we get the desired property for $P$.

Lemma 4.9. Fix a Lusztig datum $\mathbf{a}=\left(a_{k}, \lambda_{k}, a^{k}\right)$, a system of diagonals $S$, and

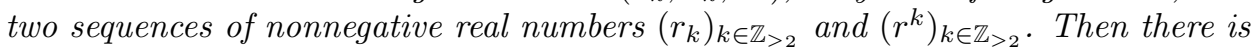
a decorated GGMS polytope $Q$ of type $S$ with right Lusztig data a such that

- for each $\left(\bar{\tau}^{k}, \tau^{k-1}\right) \in S, \mu^{k-1}-\bar{\mu}^{k}=r^{k} \alpha_{1}$,

- for each $\left(\tau^{k}, \bar{\tau}^{k-1}\right) \in S, \bar{\mu}^{k-1}-\mu^{k}=r^{k} \alpha_{0}$,

- for each $\left(\bar{\tau}_{k}, \tau_{k-1}\right) \in S, \bar{\mu}_{k}-\mu_{k-1}=r_{k} \alpha_{0}$,

- for each $\left(\tau_{k}, \bar{\tau}_{k-1}\right) \in S, \mu_{k}-\bar{\mu}_{k-1}=r_{k} \alpha_{1}$,

if and only if the following conditions hold:

(i) If $\left(\bar{\tau}_{k}, \tau_{k-1}\right),\left(\tau_{k+1}, \bar{\tau}_{k}\right) \in S$, then $r_{k}=(k-1) a_{k}+k a_{k+1}$ and $r_{k+1}=$ $k a_{k}+(k+1) a_{k+1}$.

(ii) If $\left(\bar{\tau}_{k}, \tau_{k-1}\right),\left(\bar{\tau}_{k+1}, \tau_{k}\right) \in S$, then $r_{k+1}=r_{k}+2 a_{k}$.

(iii) If $\left(\tau_{k}, \bar{\tau}_{k-1}\right),\left(\tau_{k+1}, \bar{\tau}_{k}\right) \in S$, then $r_{k+1}=r_{k}+2 a_{k+1}$.

(iv) If $\left(\tau_{k}, \bar{\tau}_{k-1}\right),\left(\bar{\tau}_{k+1}, \tau_{k}\right) \in S$, then $\frac{k-1}{k} \leq \frac{r_{k}}{r_{k+1}} \leq \frac{k}{k+1}$.

(v) If $\left(\bar{\tau}^{k}, \tau^{k-1}\right),\left(\tau^{k+1}, \bar{\tau}^{k}\right) \in S$, then $r^{k}=(k-1) a^{k}+k a^{k+1}$ and $r^{k+1}=$ $k a^{k}+(k+1) a^{k+1}$

(vi) If $\left(\bar{\tau}^{k}, \tau^{k-1}\right),\left(\bar{\tau}^{k+1}, \tau^{k}\right) \in S$, then $r^{k+1}=r^{k}+2 a^{k}$. 
(vii) If $\left(\tau^{k}, \bar{\tau}^{k-1}\right),\left(\tau^{k+1}, \bar{\tau}^{k}\right) \in S$, then $r^{k+1}=r^{k}+2 a^{k+1}$.

(viii) If $\left(\tau^{k}, \bar{\tau}^{k-1}\right),\left(\bar{\tau}^{k+1}, \tau^{k}\right) \in S$, then $\frac{k-1}{k} \leq \frac{r^{k}}{r^{k+1}} \leq \frac{k}{k+1}$.

Proof. The Lusztig data specifies the right side of $Q$. Furthermore, each diagonal in $S$, along with its length as specified by $d_{j}$ or $d^{j}$, gives a vertex on the left side. To see if there is a corresponding GGMS polytope $Q$, one must check that:

(i) If the same $\bar{\mu}_{k}$ has been specified in two ways, those ways agree.

(ii) Whenever $\bar{\mu}_{k-1}, \bar{\mu}_{k}$ have both been specified, then $\bar{\mu}_{k}-\bar{\mu}_{k-1}$ is a nonnegative multiple of $\alpha_{0}+(k-1) \delta$.

(iii) If $\bar{\mu}_{k-1}, \bar{\mu}_{k+1}$ have both been specified, there is a way to specify $\bar{\mu}_{k}$ such that $\bar{\mu}_{k}-\bar{\mu}_{k-1}$ and $\bar{\mu}_{k+1}-\bar{\mu}_{k}$ are nonnegative multiples of $\alpha_{0}+(k-1) \delta$ and $\alpha_{0}+k \delta$, respectively;

and similarly for the upper vertices. Translating, we get exactly the conditions given.

Remark 4.10. The conditions in Lemma 4.9 determine the vertices $\bar{\mu}_{k}$ and $\bar{\mu}^{k}$ of $Q$, but not the partition $\bar{\lambda}$ that appears in the left Lusztig data. However, there are two cases where $\bar{\lambda}$ can be defined:

- If either for all sufficiently large $k,\left(\bar{\tau}^{k}, \tau^{k-1}\right) \in S$ and $\left(\tau_{k}, \bar{\tau}_{k-1}\right) \in S$, or for all sufficiently large $k,\left(\tau^{k}, \bar{\tau}^{k-1}\right) \in S$ and $\left(\bar{\tau}_{k}, \tau_{k-1}\right) \in S$, then one may set $\bar{\lambda}=\lambda$.

- If for all sufficiently large $k,\left(\tau^{k}, \bar{\tau}^{k-1}\right) \in S$ and $\left(\tau_{k}, \bar{\tau}_{k-1}\right) \in S$, then one defines $\bar{\lambda}$ by adding a single part to $\lambda$; because of the conditions $\mu^{\infty}-\mu_{\infty}=|\lambda| \delta$ and $\bar{\mu}^{\infty}-\bar{\mu}_{\infty}=|\bar{\lambda}| \delta$, this part is necessarily equal to the width of $Q$.

The action of the lowering operator $f_{0}$ on $\mathcal{M V}$ can be concretely described, as shown in the following proposition (see also Figure 51).

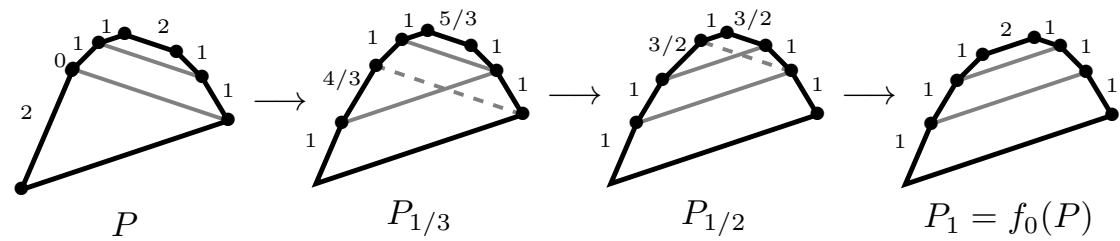

Figure 5. Applying $f_{0}$ to an MV polytope. By Proposition 4.11, $f_{0}$ only affects the part of the polytope above the top active $\alpha_{1}$ diagonal, so that is all we draw. In each figure, the active diagonals used to continue the procedure are shown in grey. The dashed diagonals are also active, but are being "replaced" by the solid diagonals that cross them.

Proposition 4.11. Let a be a Lusztig data. For $t \in\left[0, a^{1}\right]$, consider the Lusztig data $\mathbf{a}_{t}=\left(a_{1}, a_{2}, \ldots, a^{2}, a^{1}-t\right)$, and denote by $P_{t}$ the polytope with right Lusztig data $\mathbf{a}_{t}$. These polytopes $P_{t}$ can be determined starting from $P_{0}$, as follows.

Let $0 \leq t<a^{1}$. Let $d$ be the highest active diagonal in $P_{t}$ parallel to $\alpha_{1}$. Let $S$ be a system of diagonals containing $d$ such that $P$ is of type $S$. Then, for $\tau>t$ close enough to $t, P_{\tau}$ is the polytope $Q$ determined by Lemma 4.9 and Remark 4.10 , 
where the lengths of $d$ and all lower diagonals are unchanged, and the lengths of the diagonals above $d$ have all decreased by $\tau-t$. Furthermore, the conditions of Lemma 4.9 continue to hold until a new $\alpha_{1}$-diagonal above $d$ becomes active; then choose a new $S^{\prime}$ containing that diagonal, and continue. At the end of the process, the uppermost $\alpha_{1}$-diagonal $\left(\bar{\mu}^{2}, \mu^{1}\right)$ is active and the edge $\left(\bar{\mu}^{1}, \mu^{0}\right)$ coincides with this diagonal.

If in $P_{t}$ there are no active diagonals parallel to $\alpha_{1}$, then, for $\tau>t$ close enough to $t, P_{\tau}$ is the polytope $Q$ determined by Lemma 4.9 and Remark 4.10, where the lengths of all diagonals have all decreased by $\tau-t$.

Remark 4.12. There is a slight subtlety in this construction in the case when $d$ is the diagonal $\left(\bar{\mu}_{\infty}, \mu_{\infty}\right)$. Then $\bar{\lambda}$ loses its larger part and $\left(\bar{\mu}^{\infty}, \mu^{\infty}\right)$ becomes parallel to $\alpha_{1}$ as soon as $\tau$ becomes larger than $t$. Further, let $\bar{\mu}^{k}$ be the first vertex above $\bar{\mu}^{\infty}$ on the left ridge of $P_{t}$. Then for all $\tau>t$ close enough to $t$, there is an integer $r \geq k+2$ such that $\bar{\mu}^{\infty}=\bar{\mu}^{r+1}$ and $\bar{\mu}^{k+1}=\bar{\mu}^{r-1}$ in $P_{\tau}$. This $r$ depends on $\tau$ and is arbitrary large for $\tau \rightarrow t$; when $\tau$ increases, $\bar{\mu}^{r}$ moves from $\bar{\mu}^{k+1}$ to $\bar{\mu}^{\infty}$, and $r$ decrements when $\bar{\mu}^{r}$ reaches $\bar{\mu}^{\infty}$. This procedure runs smoothly as long as $r>k+2$, so the creation of a new active diagonal parallel to $\alpha_{1}$ does not happen immediately (a precise bound can be worked out from Lemma 3.15).

Remark 4.13. Let $P \in \mathcal{M} V$, and let a be the right Lusztig data of $P$. Then we have $P=P_{0}$, and Proposition 4.11 provides a concrete way to determine the polytope $f_{0}^{n}(P)=P_{n}$, for all integers $0 \leq n \leq a^{1}$. One may also describe the action of $f_{1}$ : the same algorithm can be run, but twisted by a horizontal reflection. One can run the algorithm in reverse to describe the action of the $e_{i}$. In fact, we see that the action of $e_{0}$ slowly destroys active $\alpha_{1}$ diagonals and that once there are no more active $\alpha_{1}$ diagonals, then $e_{0}$ acts by adding $\alpha_{0}$ to all the $\bar{\mu}_{k}, \bar{\mu}^{k}$ vertices (except $\bar{\mu}_{0}$ ). Proof of Proposition 4.11. Fix $0 \leq t<a^{1}$ such that $P_{t}$ has been defined, and for $\tau>t$ small enough, denote by $Q_{\tau}$ the polytope $Q$ in the statement. There is some maximal $\tau$ such that the conditions of Lemma 4.9 to ensure the existence of a GGMS polytope hold. Either condition (iv) or condition (viii) from Lemma 4.9 must fail after this point, since the other conditions are all preserved under the changes made.

If (iv) fails after $\tau$ for some $k$, then the vertices of $Q_{\tau}$ satisfy $\bar{\mu}_{k-1}=\bar{\mu}_{k}$. But then either $\left(\mu_{k+1}, \bar{\mu}_{k}\right)$ is an active diagonal, or $Q_{\tau}$ is already not an MV polytope. In the second case, there must have been a largest value of $\tau^{\prime}$ such that $Q_{\tau^{\prime}}$ was an MV polytope; at this $\tau^{\prime}$ a new active diagonal must have formed, which must be above $d$ since the polytope is unchanged below $d$. So in either case, a new active diagonal has formed above $d$. If (viii) fails a similar argument shows that, possibly at an earlier time, we had a valid MV polytope with a new active diagonal above $d$.

Thus we see that the procedure always runs. At $t=a^{1}$ one sees a polytope whose edge parallel to $\alpha_{0}$ on the right side has length 0 , from which the other claims are immediate.

Proposition 4.14. Fix $P \in \mathcal{M} V$. If $P$ has an active diagonal parallel to $\alpha_{1}$, then $f_{0} f_{0}^{*}(P)=f_{0}^{*} f_{0}(P)$ and $\varphi_{0}^{*}\left(f_{0} b\right)=\varphi_{0}^{*}(b)$. If $P$ has no such diagonal, then $f_{0}(P)=f_{0}^{*}(P)$ and $\varphi_{0}^{*}(P)>0$.

Proof. Assume $P$ has an active diagonal $d$ parallel to $\alpha_{1}$, and let $P_{\ell}$ and $P_{h}$ be the two smaller polytopes obtained by cutting along $d$, where $P_{\ell}$ is below $P_{h}$. Then 
by Proposition 4.11 $f_{0}$ and $f_{0}^{*}$ preserve the diagonal $d, f_{0}$ only affects $P_{h}$, and $f_{0}^{*}$ only affects $P_{\ell}$ (where the statements about $f_{0}^{*}$ follow by symmetry). In particular $f_{0} f_{0}^{*}(P)=f_{0}^{*} f_{0}(P)$ and $\varphi_{0}^{*}\left(f_{0} b\right)=\varphi_{0}^{*}(b)$.

If $P$ does not have an active diagonal parallel to $\alpha_{1}$, the algorithm for calculating $f_{0}(P)$ given in Proposition 4.11 must proceed without creating a new active $\alpha_{1}$ diagonal at time $t<1$ : in fact, denoting by $\bar{\mu}_{k}(t)=\bar{\mu}_{k}-t \alpha_{0}$ and $\bar{\mu}^{k}(t)=\bar{\mu}^{k}-t \alpha_{0}$ the vertices of $P_{t}$, the quantities $\left(\bar{\mu}_{k}(t)-\mu_{k+1}, \omega_{0}\right)$ and $\left(\bar{\mu}^{k}(t)-\mu^{k-1}, \omega_{0}\right)$ are of the form $c-t$ with $c$ a positive integer, so cannot vanish before $t$ reaches the value 1 . It follows that $f_{0}(P)=f_{0}^{*}(P)$. Furthermore, it follows that this quantity is nonzero, so $\varphi_{0}^{*}(P)>0$.

Remark 4.15. Proposition4.14 implies that, for all $P \in \mathcal{M} V$ and $k \geq 0, \varphi_{0}\left(\left(f_{0}^{*}\right)^{k} P\right) \leq$ $\varphi_{0}(P)$.

Lemma 4.16. For all $P \in \mathcal{M V}$ we have $\varphi_{0}(P)+\varphi_{0}^{*}(P)-\left(\alpha_{0}, w t(P)\right) \geq 0$, with equality if and only if all $\alpha_{0}$-diagonals are active in $P$.

Proof. Let $\mathbf{a}$ and $\overline{\mathbf{a}}$ the right and left Lusztig data of $P$. We first notice that

$$
\begin{aligned}
\left(\alpha_{0}, \operatorname{wt}(P)\right) & =2\left(a^{1}+a^{2}+a^{3}+\cdots-a_{1}-a_{2}-a_{3}-\cdots\right) \\
& =2\left(\bar{a}_{1}+\bar{a}_{2}+\bar{a}_{3}+\cdots-\bar{a}^{1}-\bar{a}^{2}-\bar{a}^{3}-\cdots\right) .
\end{aligned}
$$

It follows that

$$
\begin{aligned}
\varphi_{0}(P)+\varphi_{0}^{*}(P)-\left(\alpha_{0}, \mathrm{wt}(P)\right)= & \left(a_{1}-\bar{a}_{2}\right)+\left(a_{2}-\bar{a}_{3}\right)+\left(a_{3}-\bar{a}_{4}\right)+\cdots \\
& +\left(\bar{a}^{1}-a^{2}\right)+\left(\bar{a}^{2}-a^{3}\right)+\left(\bar{a}^{3}-a^{4}\right)+\cdots .
\end{aligned}
$$

In addition, the inequalities coming from the $\alpha_{0}$-diagonals (see Definition 3.4 (i) and (ii)) give

$$
0 \leq\left(\omega_{1}, \mu_{k}-\bar{\mu}_{k+1}\right)=\left(a_{1}-\bar{a}_{2}\right)+2\left(a_{2}-\bar{a}_{3}\right)+3\left(a_{3}-\bar{a}_{4}\right)+\cdots+k\left(a_{k}-\bar{a}_{k+1}\right),
$$

with equality if and only if $\left(\bar{\mu}_{k+1}, \mu_{k}\right)$ is active, and

$$
0 \leq\left(\omega_{1}, \mu^{k+1}-\bar{\mu}^{k}\right)=\left(\bar{a}^{1}-a_{2}\right)+2\left(\bar{a}^{2}-a^{3}\right)+3\left(\bar{a}^{3}-a^{4}\right)+\cdots+k\left(a^{k+1}-\bar{a}^{k}\right),
$$

with equality if and only if $\left(\mu^{k+1}, \bar{\mu}^{k}\right)$ is active. Thus,

$$
\begin{gathered}
\varphi_{0}(P)+\varphi_{0}^{*}(P)-\left(\alpha_{0}, \operatorname{wt}(P)\right)=\sum_{r=1}^{\infty}\left(a_{r}-\bar{a}_{r+1}\right)+\sum_{r=1}^{\infty}\left(\bar{a}^{r}-a^{r+1}\right) \\
=\sum_{k=1}^{\infty} \frac{1}{k(k+1)}\left(\left(\omega_{1}, \mu_{k}-\bar{\mu}_{k+1}\right)+\left(\omega_{1}, \mu^{k+1}-\bar{\mu}^{k}\right)\right) \geq 0
\end{gathered}
$$

with equality if and only if all diagonals $\left(\bar{\mu}_{k+1}, \mu_{k}\right)$ and $\left(\mu^{k+1}, \bar{\mu}^{k}\right)$ are active.

Lemma 4.17. A polytope $P \in \mathcal{M V}$ has an active diagonal parallel to $\alpha_{1}$ if and only if $\varepsilon_{0}\left(\left(f_{0}^{*}\right)^{\max }(P)\right)-\varphi_{0}^{*}(P) \geq 0$, where we use $\left(f_{0}^{*}\right)^{\max }(P)$ as shorthand for $\left(f_{0}^{*}\right)^{\varphi_{0}^{*}(P)}(P)$.

Proof. Let $P \in \mathcal{M} V$. Set $n=\varphi_{0}^{*}(P)$ and $Q=\left(f_{0}^{*}\right)^{n}(P)$. We have

$$
\begin{aligned}
\varepsilon_{0}\left(\left(f_{0}^{*}\right)^{\max }(P)\right)-\varphi_{0}^{*}(P) & =\varphi_{0}(Q)-\left(\alpha_{0}, \operatorname{wt}(Q)\right)+\varphi_{0}^{*}(P)-2 n \\
& =\varphi_{0}(Q)+\varphi_{0}^{*}(P)-\left(\alpha_{0}, \operatorname{wt}(P)\right) .
\end{aligned}
$$


Suppose first that $P$ has an active diagonal parallel to $\alpha_{1}$. Then $\varphi_{0}(P)=\varphi_{0}(Q)$, by (the proof of) Proposition 4.14. Hence using Lemma 4.16, we get

$$
\varepsilon_{0}\left(\left(f_{0}^{*}\right)^{\max }(P)\right)-\varphi_{0}^{*}(P)=\varphi_{0}(P)+\varphi_{0}^{*}(P)-\left(\alpha_{0}, \operatorname{wt}(P)\right) \geq 0,
$$

as desired.

Suppose now that all $\alpha_{1}$-diagonals are inactive in $P$. Then $n=\varphi_{0}^{*}(P)>0$, for otherwise we would have $\mu_{1}=\mu_{0}$, which would force the $\alpha_{1}$-diagonal $\left(\bar{\mu}_{2}, \mu_{1}\right)$ to be active. It then follows from Proposition 4.14 and Remark 4.15 that $\varphi_{0}(Q)=$ $\varphi_{0}\left(\left(f_{0}^{*}\right)^{n} P\right) \leq \varphi_{0}\left(f_{0}^{*} P\right)=\varphi_{0}\left(f_{0} P\right)<\varphi_{0}(P)$, and therefore

$$
\varepsilon_{0}\left(\left(f_{0}^{*}\right)^{\max }(P)\right)-\varphi_{0}^{*}(P)<\varphi_{0}(P)+\varphi_{0}^{*}(P)-\left(\alpha_{0}, \operatorname{wt}(P)\right) .
$$

Since all $\alpha_{0}$-diagonals are active in $P$ the right-hand side is 0 .

By Lemma 4.8 $\mathcal{M V}$ is a lowest weight combinatorial crystal, so to finish the proof of Theorem 4.5. we must verify the conditions of Corollary 2.7. For $i=0, j=1$, (ii) follows because $f_{0}^{*}$ only affect $\bar{a}_{1}$ (not the rest of $\overline{\mathbf{a}}$ ) and $f_{1}$ only affect $\bar{a}^{1}$. For $i=0$, (iii) and (iii) follow from Lemma 4.17 and Proposition 4.14, The other cases follow by symmetry.

\section{5. $A_{2}^{(2)}$ MV POLYTOPES}

We now describe MV polytopes for the only other rank two affine root system. One could perhaps rework the proof as in the $\widehat{\mathrm{sl}}_{2}$ case and directly prove that the MV polytopes we describe below have the desired properties. However, we find it easier to use Kashiwara similarity of crystals (Theorem 2.9), which provides an embedding of $B^{A_{2}^{(2)}}(-\infty)$ into $B^{\widehat{\mathrm{sl}}_{2}}(-\infty)$, where we use superscripts to distinguish data related to different root systems. There is a corresponding vector space isomorphism from the real spans of the $\widehat{\mathrm{sl}}_{2}$ simple roots to the real span of the $A_{2}^{(2)}$ simple roots, and essentially our $A_{2}^{(2)} \mathrm{MV}$ polytopes are the pullbacks of certain $\widehat{\mathrm{sl}}_{2} \mathrm{MV}$ polytopes under this map.

5.1. The polytopes. Recall that $A_{2}^{(2)}$ is the affine Kac-Moody algebra with symmetrized Cartan matrix

$$
\tilde{N}=\left(\begin{array}{rr}
2 & -4 \\
-4 & 8
\end{array}\right)
$$

Let $\tilde{\alpha}_{0}$ and $\tilde{\alpha}_{1}$ be the simple roots, where $\left(\tilde{\alpha}_{0}, \tilde{\alpha}_{0}\right)=2$ and $\left(\tilde{\alpha}_{1}, \tilde{\alpha}_{1}\right)=8$. The set of positive roots of $A_{2}^{(2)}$ is $\Delta_{r e}^{+} \sqcup \Delta_{i m}^{+}$, where, setting $\tilde{\delta}=2 \tilde{\alpha}_{0}+\tilde{\alpha}_{1}$,

$$
\begin{aligned}
\Delta_{r e}^{+} & =\left\{\tilde{\alpha}_{0}+k \tilde{\delta}, \tilde{\alpha}_{1}+2 k \tilde{\delta}, \tilde{\alpha}_{0}+\tilde{\alpha}_{1}+k \tilde{\delta}, 2 \tilde{\alpha}_{0}+(2 k+1) \tilde{\delta} \mid k \geq 0\right\} \quad \text { and } \\
\Delta_{i m}^{+} & =\{k \delta \mid k \geq 1\} .
\end{aligned}
$$

Here $\Delta_{r e}^{+}$is the set of real positive roots and $\Delta_{i m}^{+}$is the imaginary positive roots. 
These can be drawn as

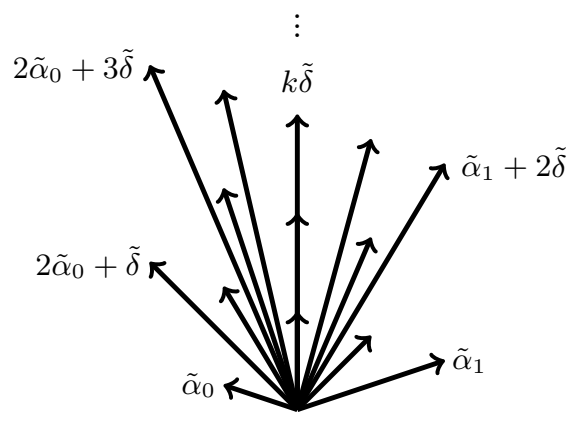

Definition 5.1. Label the real roots for $A_{2}^{(2)}$ by

$$
r_{k}=\left\{\begin{array}{ll}
\tilde{\alpha}_{1}+(k-1) \tilde{\delta} & \text { if } k \text { is odd, } \\
\tilde{\alpha}_{0}+\tilde{\alpha}_{1}+\frac{k-2}{2} \tilde{\delta} & \text { if } k \text { is even, }
\end{array} \quad r^{k}= \begin{cases}\tilde{\alpha}_{0}+\frac{k-1}{2} \tilde{\delta} & \text { if } k \text { is odd, } \\
2 \tilde{\alpha}_{0}+(k-1) \tilde{\delta} & \text { if } k \text { is even }\end{cases}\right.
$$

Remark 5.2. In the language of [2], $r_{1} \prec r_{2} \prec \cdots \prec \delta \prec \cdots \prec r^{2} \prec r^{1}$ and $r^{1} \prec r^{2} \prec \cdots \prec \delta \prec \cdots \prec r_{2} \prec r_{1}$ are the two possible convex orders.

Definition 5.3. An $A_{2}^{(2)}$ GGMS polytope is a convex polytope in $\operatorname{span}_{\mathbb{R}}\left\{\tilde{\alpha}_{0}, \tilde{\alpha}_{1}\right\}$ such that all edges are parallel to roots. Such a polytope is called integral if all vertices lie in $\operatorname{span}_{\mathbb{Z}}\left\{\tilde{\alpha}_{0}, \tilde{\alpha}_{1}\right\}$.

As in the $\widehat{s l}_{2}$ case, we can record a GGMS polytope by recording the positions of each vertex. The vertices are labeled $\mu_{k}, \mu^{k}, \bar{\mu}_{k}, \bar{\mu}^{k}, \mu_{\infty}, \mu^{\infty}, \bar{\mu}_{\infty}, \bar{\mu}^{\infty}$, where e.g. $\mu_{k}$ records the vertex after the edge in the direction $r_{k}$ moving up the polytope on the right side, and $\bar{\mu}^{k-1}$ records the vertex after the edge in the direction $r_{k}$ moving up the polytope on the left side. We also associate to a GGMS polytope $P$ the data $\left\{a_{k}, a^{k}, \bar{a}_{k}, \bar{a}^{k}\right\}$ where e.g. $\mu_{k}-\mu_{k-1}=a_{k} r_{k}$.

Definition 5.4. A decorated $A_{2}^{(2)}$ GGMS polytope is a GGMS polytope along with a choice of two sequences $\lambda=\left(\lambda_{1} \geq \lambda_{2} \geq \cdots\right)$ and $\bar{\lambda}=\left(\bar{\lambda}_{1} \geq \bar{\lambda}_{2} \geq \cdots\right)$ of positive real numbers such that $\mu^{\infty}-\mu_{\infty}=\left(\lambda_{1}+\lambda_{2}+\cdots\right) \tilde{\delta}$ and $\bar{\mu}^{\infty}-\bar{\mu}_{\infty}=\left(\bar{\lambda}_{1}+\bar{\lambda}_{2}+\cdots\right) \tilde{\delta}$ and, for all large enough $N, \lambda_{N}=\bar{\lambda}_{N}=0$. Such a polytope is called integral if the underlying GGMS polytope is integral and all $\lambda_{k}, \bar{\lambda}_{k}$ are integers.

Definition 5.5. The right Lusztig data of a decorated $A_{2}^{(2)}$ GGMS polytope is the data $\mathbf{a}=\left(a_{k}, \lambda_{k}, a^{k}\right)_{k \in \mathbb{N}}$. The left Lusztig data is $\overline{\mathbf{a}}=\left(\bar{a}_{k}, \bar{\lambda}_{k}, \bar{a}^{k}\right)_{k \in \mathbb{N}}$.

Let $\tilde{\omega}_{0}^{\vee}$ and $\tilde{\omega}_{1}^{\vee}$ be a choice of fundamental coweights (i.e. any choice of elements in weight space satisfying $\left(\tilde{\omega}_{i}^{\vee}, \tilde{\alpha}_{j}\right)=\delta_{i, j}$, where we are using the bilinear form to identify weight space and coweight space).

Definition 5.6. An $A_{2}^{(2)}$ MV polytope is a decorated $A_{2}^{(2)}$ GGMS polytope such that

(i) For each $k \geq 1,\left(\bar{\mu}_{k}-\mu_{k-1}, \tilde{\omega}_{1}^{\vee}\right) \leq 0$ and $\left(\mu_{k}-\bar{\mu}_{k-1}, \tilde{\omega}_{0}^{\vee}\right) \leq 0$, with at least one of these being an equality.

(ii) For each $k \geq 1,\left(\bar{\mu}^{k}-\mu^{k-1}, \tilde{\omega}_{0}^{\vee}\right) \geq 0$ and $\left(\mu^{k}-\bar{\mu}^{k-1}, \tilde{\omega}_{1}^{\vee}\right) \geq 0$, with at least one of these being an equality.

(iii) If $\left(\mu_{\infty}, \bar{\mu}_{\infty}\right)$ and $\left(\mu^{\infty}, \bar{\mu}^{\infty}\right)$ are parallel then $\lambda=\bar{\lambda}$. Otherwise, one is obtained from the other by removing a part of size $\left(\mu_{\infty}-\bar{\mu}_{\infty}, \tilde{\alpha}_{1}\right) / 8$.

(iv) $\lambda_{1}, \bar{\lambda}_{1} \leq\left(\mu_{\infty}-\bar{\mu}_{\infty}, \tilde{\alpha}_{1}\right) / 8$. 
$P$ is called integral if it is integral as a decorated GGMS polytope. We denote by $\mathcal{M} V^{A_{2}^{(2)}}$ the set of integral $A_{2}^{(2)} \mathrm{MV}$ polytopes up to translation.

5.2. Proof that they realize $B^{A_{2}^{(2)}}(-\infty)$. Let $V=\operatorname{span}_{\mathbb{R}}\left(\alpha_{0}, \alpha_{1}\right)$ and $\tilde{V}=$ $\operatorname{span}_{\mathbb{R}}\left(\tilde{\alpha}_{0}, \tilde{\alpha}_{1}\right)$. Consider the map $\gamma: V \rightarrow \tilde{V}$ defined by $\alpha_{0} \mapsto \tilde{\alpha}_{0}, \alpha_{1} \mapsto \tilde{\alpha}_{1} / 2$. This sends each positive $\widehat{s l}_{2}$ root to a multiple of a positive $A_{2}^{(2)}$ root (the multiple is either 1 or $1 / 2$ ), and is a bijection on root directions (i.e. roots up to scalar). It follows that $\gamma$ defines a bijection from (not necessarily integral) $\widehat{\mathrm{sl}}_{2}$ GGMS polytopes to $A_{2}^{(2)}$ GGMS polytopes. Extend this map to a map of decorated GGMS polytopes by $\gamma(\lambda)_{k}=\lambda_{k} / 2, \gamma(\bar{\lambda})_{k}=\bar{\lambda}_{k} / 2$.

Lemma 5.7. $\gamma^{-1}\left(\mathcal{M} V^{A_{2}^{(2)}}\right) \subset \mathcal{M} V^{\widehat{\mathrm{sl}}_{2}}$.

Proof. This follows from the observation that $\gamma^{-1}$ of a lattice point in $\operatorname{span}_{\mathbb{R}}\left(\tilde{\alpha}_{0}, \tilde{\alpha}_{1}\right)$ is a lattice point in $\operatorname{span}_{\mathbb{R}}\left(\alpha_{0}, \alpha_{1}\right)$.

Lemma 5.8. For $P \in \mathcal{M} V^{\widehat{\mathrm{s}}_{2}}, \gamma(P) \in \mathcal{M} V^{A_{2}^{(2)}}$ if and only if any of the following three equivalent conditions hold, where $\mathbf{a}$ and $\overline{\mathbf{a}}$ are the right and left Lusztig data for $P$ :

(i) $a_{k}, \bar{a}^{k}$ are even whenever $k$ is odd, $a^{k}, \bar{a}_{k}$ are even whenever $k$ is even, all $\lambda_{k}, \bar{\lambda}_{k}$ are even.

(ii) $a_{k}$ is even whenever $k$ is odd, $a^{k}$ is even whenever $k$ is even, all $\lambda_{k}$ are even.

(iii) $\bar{a}_{k}$ is even whenever $k$ is even, $\bar{a}^{k}$ is even whenever $k$ is odd, all $\bar{\lambda}_{k}$ are even.

Proof. First we check that the three conditions are equivalent. Clearly (ii) implies (iii) and (iii). To see that (iii) implies (ii), recall that we can cut $P$ into quadrilaterals by cutting along active diagonals, and that these quadrilaterals are themselves all MV polytopes. The quadrilaterals that appear are all shown in Figure 2 and it is straightforward to see that, for each of these, the conditions on a from (iii) imply the conditions from (ii) on the data $\overline{\mathbf{a}}$. Gluing these together shows that (iii) implies (ii) for $P$. Showing (iii) implies (ii) is similar.

Condition (1i) is exactly the condition that $\gamma$ sends each edge to a multiple of an $A_{2}^{(2)}$ root, or equivalently that $\gamma(P) \in \mathcal{M} V^{A_{2}^{(2)}}$.

The symmetrized Cartan matrices $N$ for $\widehat{\mathrm{sl}}_{2}$ and $\tilde{N}$ for $A_{2}^{(2)}$ satisfy $\tilde{N}=D N D$, where $D=\operatorname{diag}\{1,2\}$. Hence by Theorem 2.9 there is a unique embedding $S$ : $B^{A_{2}^{(2)}}(-\infty) \rightarrow B^{\widehat{s l}_{2}}(-\infty)$ such that $S\left(b_{-}^{N^{\prime}}\right)=b_{-}^{N}$, and, for all $b \in B^{A_{2}^{(2)}}(-\infty)$, $S\left(e_{0} b\right)=e_{0} S(b)$ and $S\left(e_{1} b\right)=e_{1}^{2} S(b)$.

Lemma 5.9. Let $\mathcal{M} V^{S}=\left\{P_{S(b)}: b \in B^{A_{2}^{(2)}}(-\infty)\right\}$. Then $\mathcal{M} V^{S}=\gamma^{-1} \mathcal{M} V^{A_{2}^{(2)}}$.

Proof. Using Lemma 5.8, one sees that $\gamma^{-1}\left(\mathcal{M} V^{A_{2}^{(2)}}\right)$ is closed under the operators $e_{0}, f_{0}, e_{1}^{2}, f_{1}^{2}$. Furthermore, it is clear that, for any nontrivial $P \in \gamma^{-1}\left(\mathcal{M} V_{2}^{A_{2}^{(2)}}\right)$, either $f_{0}(P) \neq \emptyset$ or $f_{1}^{2}(P) \neq \emptyset$. The claim now follows from the defining conditions on $S$ and the fact that $B^{A_{2}^{(2)}}(-\infty)$ is generated under the action of $e_{0}$ and $e_{1}$ by its lowest weight element. 
Theorem 5.10. There is a unique $\tilde{P} \in \mathcal{M} V_{2}^{A_{2}^{(2)}}$ for each choice of integral right Lusztig data. Furthermore, $\mathcal{M} V_{2}^{A_{2}^{(2)}}$ along with the crystal operators defined as in Definition 4.1, is a copy of $B^{A_{2}^{(2)}}(-\infty)$.

Proof. For $b \in B^{A_{2}^{(2)}}(-\infty)$, let $\tilde{P}_{b}=\gamma\left(P_{S(b)}\right)$. Lemma 5.9 shows that $b \rightarrow \tilde{P}_{b}$ is a bijection between $B^{A_{2}^{(2)}}(-\infty)$ and $\mathcal{M} V_{2}^{A_{2}^{(2)}}$. All the required properties now follow from the corresponding results about $\widehat{\mathrm{sl}}_{2} \mathrm{MV}$ polytopes.

\section{ACKnowledgments}

The authors would like to thank Dinakar Muthiah for helpful conversations and the anonymous referee for pointing out a few inaccuracies and suggesting numerous improvements.

\section{RefERENCES}

[1] Jared E. Anderson, A polytope calculus for semisimple groups, Duke Math. J. 116 (2003), no. 3, 567-588, DOI 10.1215/S0012-7094-03-11636-1. MR.1958098 (2004a:20047)

[2] Pierre Baumann, Joel Kamnitzer, and Peter Tingley. Affine Mirković-Vilonen polytopes. Preprint. arXiv:1110.3661.

[3] Jonathan Beck, Vyjayanthi Chari, and Andrew Pressley, An algebraic characterization of the affine canonical basis, Duke Math. J. 99 (1999), no. 3, 455-487, DOI 10.1215/S0012-709499-09915-5. MR.1712630 (2000g:17013)

[4] Ilaria Damiani, A basis of type Poincaré-Birkhoff-Witt for the quantum algebra of $\widehat{\mathrm{sl}}(2)$, J. Algebra 161 (1993), no. 2, 291-310, DOI 10.1006/jabr.1993.1220. MR.1247357 (94k:17021)

[5] Thomas Rough Dunlap II, Combinatorial representation theory of affine sl2 via polytope calculus, ProQuest LLC, Ann Arbor, MI, 2010. Thesis (Ph.D.)-Northwestern University. MR.2736801

[6] Jin Hong and Seok-Jin Kang, Introduction to quantum groups and crystal bases, Graduate Studies in Mathematics, vol. 42, American Mathematical Society, Providence, RI, 2002. MR:1881971 (2002m:17012)

[7] Victor G. Kac, Infinite-dimensional Lie algebras, 3rd ed., Cambridge University Press, Cambridge, 1990. MR:1104219 (92k:17038)

[8] Joel Kamnitzer, The crystal structure on the set of Mirković-Vilonen polytopes, Adv. Math. 215 (2007), no. 1, 66-93, DOI 10.1016/j.aim.2007.03.012. MR2354986 (2009a:17021)

[9] Joel Kamnitzer, Mirković-Vilonen cycles and polytopes, Ann. of Math. (2) 171 (2010), no. 1, 245-294, DOI 10.4007/annals.2010.171.245. MR2630039 (2011g:20070)

[10] Masaki Kashiwara, The crystal base and Littelmann's refined Demazure character formula, Duke Math. J. 71 (1993), no. 3, 839-858, DOI 10.1215/S0012-7094-93-07131-1. MR1240605 (95b:17019)

[11] Masaki Kashiwara, On crystal bases, Representations of groups (Banff, AB, 1994), CMS Conf. Proc., vol. 16, Amer. Math. Soc., Providence, RI, 1995, pp. 155-197. MR.1357199 (97a:17016)

[12] Masaki Kashiwara, Similarity of crystal bases, Lie algebras and their representations (Seoul, 1995), Contemp. Math., vol. 194, Amer. Math. Soc., Providence, RI, 1996, pp. 177-186, DOI 10.1090/conm/194/02393. MR1395599 (97g:17013)

[13] Masaki Kashiwara and Yoshihisa Saito, Geometric construction of crystal bases, Duke Math. J. 89 (1997), no. 1, 9-36, DOI 10.1215/S0012-7094-97-08902-X. MR1458969 (99e:17025)

[14] George Lusztig, Introduction to quantum groups, Progress in Mathematics, vol. 110, Birkhäuser Boston Inc., Boston, MA, 1993. MR1227098 (94m:17016)

[15] Dinakar Muthiah, Double MV cycles and the Naito-Sagaki-Saito crystal, Adv. Math. 240 (2013), 268-290. MR.3046309

[16] Dinakar Muthiah and Peter Tingley, Affine PBW bases and $M V$ polytopes in rank 2, Selecta Mathematica, published on line January 24, 2013, DOI 10.1007/s0029-012-0117-z.

[17] Satoshi Naito, Daisuke Sagaki, and Yoshihisa Saito, Toward Berenstein-Zelevinsky data in affine type A, I: Construction of affine analogs, Algebraic groups and quantum groups, 143184, Contemp. Math., 565, Amer. Math. Soc., Providence, RI, 2012. MR2932426 
Institut de Recherche Mathématique Avancée, Université de Strasbourg et CNRS, 7 rue René Descartes, 67084 Strasbourg Cedex, France

E-mail address: p.baumann@unistra.fr

Einstein Institute of Mathematics, The Hebrew University of Jerusalem, Jerusalem, 91904, ISRAEL

E-mail address: tdunlap@umich.edu

Department of Mathematics, University of Toronto, Toronto, On, M5S 2E4 Canada

E-mail address: jkamnitz@math.toronto.edu

Department of Mathematics and Statistics, Loyola University, Chicago, Illinois 60660

E-mail address: ptingley@luc.edu 